\title{
Turbulent mixing driven by spherical implosions. Part 2. Turbulence statistics
}

\author{
M. Lombardini , D. I. Pullin and D. I. Meiron \\ Graduate Aerospace Laboratories, California Institute of Technology, Pasadena, CA 91125, USA
}

(Received 12 June 2013; revised 29 January 2014; accepted 20 March 2014;

first published online 28 April 2014)

We present large-eddy simulations (LES) of turbulent mixing at a perturbed, spherical interface separating two fluids of differing densities and subsequently impacted by a spherically imploding shock wave. This paper focuses on the differences between two fundamental configurations, keeping fixed the initial shock Mach number $\approx 1.2$, the density ratio (precisely $\left|A_{0}\right| \approx 0.67$ ) and the perturbation shape (dominant spherical wavenumber $\ell_{0}=40$ and amplitude-to-initial radius of $3 \%$ ): the incident shock travels from the lighter fluid to the heavy one, or inversely, from the heavy to the light fluid. In Part 1 (Lombardini, M., Pullin, D. I. \& Meiron, D. I., J. Fluid Mech., vol. 748, 2014, pp. 85-112), we described the computational problem and presented results on the radially symmetric flow, the mean flow, and the growth of the mixing layer. In particular, it was shown that both configurations reach similar convergence ratios $\approx 2$. Here, turbulent mixing is studied through various turbulence statistics. The mixing activity is first measured through two mixing parameters, the mixing fraction parameter $\Theta$ and the effective Atwood ratio $A_{e}$, which reach similar late time values in both light-heavy and heavy-light configurations. The Taylor-scale Reynolds numbers attained at late times are estimated at approximately 2000 in the light-heavy case and 1000 in the heavy-light case. An analysis of the density self-correlation $b$, a fundamental quantity in the study of variable-density turbulence, shows asymmetries in the mixing layer and non-Boussinesq effects generally observed in high-Reynoldsnumber Rayleigh-Taylor (RT) turbulence. These traits are more pronounced in the light-heavy mixing layer, as a result of its flow history, in particular because of RT-unstable phases (see Part 1). Another measure distinguishing light-heavy from heavy-light mixing is the velocity-to-scalar Taylor microscales ratio. In particular, at late times, larger values of this ratio are reported in the heavy-light case. The late-time mixing displays the traits some of the traits of the decaying turbulence observed in planar Richtmyer-Meshkov (RM) flows. Only partial isotropization of the flow (in the sense of turbulent kinetic energy (TKE) and dissipation) is observed at late times, the Reynolds normal stresses (and, thus, the directional Taylor microscales) being anisotropic while the directional Kolmogorov microscales approach isotropy. A spectral analysis is developed for the general study of statistically isotropic turbulent fields on a spherical surface, and applied to the present flow. The resulting angular power spectra show the development of an inertial subrange approaching a Kolmogorov-like $-5 / 3$ power law at high wavenumbers, similarly to the scaling obtained in planar geometry. It confirms the findings of Thomas \& Kares (Phys. Rev. Lett., vol. 109, 2012, 075004) at higher convergence ratios and indicates that the turbulent scales do not seem to feel the effect of the spherical mixing-layer curvature.

Key words: compressible turbulence, shock waves, turbulent mixing

$\dagger$ Email address for correspondence: manuel@ cattech.edu 


\section{Introduction}

Molecular mixing as a consequence of stirring by fluid motion is a process of fundamental importance in a myriad of applications. Variable-density mixing, i.e. mixing between fluids of differing microscopic densities, which is encountered for instance in geophysical and astrophysical flows, combustion and fluidized beds, is often driven by acceleration, e.g. gravity. When the local density gradient and the pressure gradient generated by the acceleration field are misaligned, baroclinic vorticity is generated and perturbations of the initial density layer can grow nonlinearly and lead to turbulent mixing. In a constant acceleration environment, this is known as the Rayleigh-Taylor (RT) instability (Taylor 1950). Its impulsive analogue, e.g. when a density interface is accelerated by the passage of a shock wave, is referred to as the Richtmyer-Meshkov (RM) instability (Richtmyer 1960; Meshkov 1969). Unlike RT flows, which are unstable only when the density gradient is in the opposite direction to the acceleration, i.e. $\nabla p \cdot \nabla \rho<0$ (e.g. heavy fluid atop light fluid in a gravitational field), RM perturbations grow whether the incident shock wave propagates from a light to a heavy fluid $(\nabla p \cdot \nabla \rho<0)$ or from a heavy to a light fluid $(\nabla p \cdot \nabla \rho>0)$.

The study of baroclinic instabilities in curved geometries, in particular when the mean isopycnic and isobaric cylindrical/spherical surfaces are concentric to each other, is relevant to problems spanning a wide range of scales, from supernovae collapse (Joggerst, Almgren \& Woosley 2010) to explosive detonation (Balakrishnan \& Menon 2011) and inertial confinement fusion (ICF) (Welser-Sherrill et al. 2008). In these implosion/explosion-driven flows, density inhomogeneities are not only RM unstable, but also radially accelerated/decelerated and therefore subject to RT instabilities as they geometrically contract or expand, as discussed in Part 1 (Lombardini, Pullin \& Meiron 2014). We have also seen that a spherical interface initially impacted by a converging shock is reshocked multiple times, whether the interface is initially processed in a light-to-heavy fashion or vice versa. The turbulent mixing observed in RM flows with reshock has been studied in light-heavy and heavy-light configurations in planar geometry (Lombardini et al. 2011), but little work has been done in spherical geometry, for two-dimensional (2D) axisymmetric flows (Glimm et al. 2002) or fully three-dimensional (3D) flows (Youngs \& Williams 2008). Thomas \& Kares (2012) initialized a 3D simulation in an octant from a 2D flow: first, a 2D axisymmetric problem with azimuthal perturbations in a quadrant was run up to after the first reshock; at that point the solution was rotated into a 3D octant and continued to late times. Youngs \& Williams (2008) and Thomas \& Kares (2012) used numerically diffusive schemes to capture the shock and model the turbulent dissipation.

In Part 1 we described a different approach to that of Youngs \& Williams (2008) to simulate the 3D turbulent mixing driven by spherical implosions. We performed Cartesian-grid based large-eddy simulations (LES) of the two-component flow using an explicit subgrid-scale (SGS) model and a low-numerical dissipation advection scheme applied in the turbulent but smooth regions. The computational domain considered is either an octant of a sphere or a full sphere. The initial converging shock is generated as a self-similar, radially symmetric solution of the Euler equation. Spherical harmonics are used to construct the pre-shock interfacial perturbations. We continue here the investigation of two canonical configurations: the light fluid enclosed by the spherical interface, or vice versa, as summarized in table 1 . While Part 1 has focused on the importance of understanding the mean flow for the study of the mixing-layer growth, various turbulence statistics are presented here. In $\S 2$, we analyse the mixing through various measures, with a particular focus on variable-density effects through the density self-correlation $b$ defined below. Some 


$$
\text { (a) } \begin{aligned}
\text { Air } & \rightarrow \mathrm{SF}_{6} & (b) \mathrm{SF}_{6} & \rightarrow \text { air } \\
A_{0} & \approx 0.667 & A_{0} & \approx-0.667 \\
M_{S_{0}} & =1.2 & M_{S_{0}} & \approx 1.222
\end{aligned}
$$

TABLE 1. Table of runs for the light-heavy and heavy-light configurations considered indicating pre-shock Atwood ratios and incident shock Mach number at impact.

characteristics of the turbulence such as Taylor and Kolmogorov microscales, based on turbulent kinetic energy (TKE) and dissipation, are discussed in $\$ 3$. Section 4 details how to perform a spectral analysis of turbulent fields on a spherical surface, which is used to represent power spectra of the late-time turbulent mixing as well as to build the initial perturbation field presented in Part 1.

\section{Mixing statistics}

In this section, we study the evolution of some important statistics in variabledensity flows: the mixing quantities $\Theta(t)$ and $A_{e}(t)$, and the density self-correlation $b(r, t)$. These three quantities are computed from spherical surface averages defined below. We recall that fluctuations from a surface average $\langle Q\rangle$ and from a Favre-like surface average $\widetilde{Q} \equiv\langle\rho Q\rangle /\langle\rho\rangle$, where $\rho$ is the density of the mixture, are given by

$$
Q^{\prime}(r, \theta, \phi ; t)=Q(r, \theta, \phi ; t)-\langle Q\rangle(r, t), \quad Q^{\prime \prime}(r, \theta, \phi ; t)=Q(r, \theta, \phi ; t)-\tilde{Q}(r, t) .
$$

We insist that these are surface-averaged statistics performed on LES data obtained by solving the Favre-filtered Navier-Stokes equations. For clarity, we have omitted bars and tildes that usually denote the filtering operations.

\subsection{Mixing quantities $\Theta$ and $A_{e}$}

Consider that the amount of mixed fluid results from the passive chemical equilibrium between light and heavy fluids. The mass fraction of product is

$$
\frac{[1-\langle\psi\rangle(r, t)]\langle\psi\rangle(r, t)}{\left[1-\langle\psi\rangle\left(r_{c}, t\right)\right]\langle\psi\rangle\left(r_{c}, t\right)},
$$

with $\langle\psi\rangle\left(r_{c}, t\right)=0.5$, and the mixing-layer width $\delta$ at time $t$, defined by

$$
\delta(t)=\int_{0}^{\infty} 4\langle\psi\rangle(1-\langle\psi\rangle) \mathrm{d} r,
$$

can then be interpreted as a product thickness that would result if the entrained fluids were perfectly mixed in the directions $\theta$ and $\phi$. Using the scalar field $Y \equiv 2 \psi-1$, the ratio

$$
\Theta(t)=\frac{\int_{0}^{\infty}\left(1-\left\langle Y^{2}\right\rangle\right) \mathrm{d} r}{\int_{0}^{\infty}\left(1-\langle Y\rangle^{2}\right) \mathrm{d} r} \equiv \frac{\mathscr{P}(t)}{\delta(t)},
$$

characterizes the relative amount of molecularly mixed fluid within the mixing layer, i.e. the total chemical product formed $(\mathscr{P})$ relative to the maximum chemical 
product or product that would be formed if all entrained fluid were completely mixed within the layer-centre spherical surface $(\delta)$, as defined by Youngs (1994). Here, molecular mixing is a surrogate for a chemical reaction. Another mixing variable is the effective Atwood ratio $A_{e}$, which is defined as the turbulent density intensity $\left\langle\rho^{\prime 2}\right\rangle^{1 / 2} /\langle\rho\rangle$ evaluated at the layer-centre surface:

$$
A_{e}(t)=\frac{\sqrt{\left\langle\rho^{\prime 2}\right\rangle\left(r_{c}, t\right)}}{\langle\rho\rangle\left(r_{c}, t\right)} .
$$

The quantities $\Theta$ and $A_{e}$ complement statistics based solely on $\langle Y\rangle$ (e.g. $\delta$ ) that cannot distinguish between fluid locally mixed for example at a fraction $Y(x, t)=0$ and unmixed fluid in equal proportions in a particular spherical surface for which $\langle Y\rangle(r, t)=0$. According to the definitions of $\Theta$ and $A_{e}$, completely mixed fluid (i.e. homogeneity across the layer-centre spherical surface) is characterized by $\Theta=1$ and $A_{e} / A=0$, whereas $\Theta=0$ and $A_{e} / A=1$ corresponds to complete segregation (i.e. immiscible case).

Figure 1 compares the evolution of the maximum chemical product $\delta(t)$ with that of the total chemical product $\mathscr{P}(t)$. We note that, in the light-heavy case, $\mathscr{P}$ increases by a factor of approximately 5 in the time stretch $0.6 \lesssim \Delta u t / R_{0} \lesssim 1.1$ following the first reshock and an RT-unstable window, whereas $\delta$ rises by an even larger factor (approximately 6-fold) in that window. This suggests not only an intense mixing activity, but an even more important growth of larger, entrained eddies which are responsible for an actual decline of $\Theta$ immediately following the reshock-induced phase reversal $\left(\Delta u t / R_{0} \approx 0.6\right)$ until $\Delta u t / R_{0} \approx 0.8$ (figure $\left.2 a\right)$. From then, molecular mixing sustained by RT potential energy becomes predominant and $\Theta$ starts increasing while $A_{e}$ decreases. At late times, the mixing slows down as the mixing layer keeps growing. Ultimately, $\Theta$ and $A_{e} / A$ reach the values $\approx 0.8$ and 0.6 , respectively. In the heavy-light configuration, the increase of $\Theta$ and $A_{e}$ from $\Delta u t / R_{0} \gtrsim 0.12$ apparent in figure 2(b) confirms that the main contributing factor to the mixing activity is the RT unstable phase, when the interface decelerates inward prior to the first reshock (see figure $14 b$ of Part 1). The two gases mix at the final levels $\Theta \approx 0.8$ and $A_{e} / A \approx 0.6$ comparable to the values obtained in the light-heavy configuration, even though the turbulent mixing follows from a different flow history. For comparison, the planar RT-unstable mixing simulations of Cook, Cabot \& Miller (2004), which also employed the same gas combination and an initial perturbation spectrum of the Gaussian type but with different peak wavenumber and standard deviation, achieved $\Theta \approx 0.78$ and $A_{e} / A \approx 0.48$.

\subsection{Density self-correlation $b$}

We have seen in appendix A of Part 1 that the mixing layer grows asymmetrically, with the spikes and bubbles rising at different mean radial velocities. This is not only a purely geometric effect, but also a general feature of mixing at high $A$, as opposed to Boussinesq flows in which densities are close in value and the mixing layer remains symmetrical around the centreline. In variable-density, multicomponent flows at vastly different densities, the diffusive mass flux strongly depends on the mass fractions and the density field, which is itself a function of the mass fractions. This leads to nonlinear phenomena that are not seen in constant-density mixing. For instance, Livescu \& Ristorcelli (2009) comment how, in variable-density flows, light fluid mixing into heavy fluid operates differently than heavy into light mixing, and how this introduces a new source of skewness of the probability density function.

A general feature of mixing at high $A$ is the tight coupling between the density and velocity fields that requires a careful study of turbulent statistics such as the 

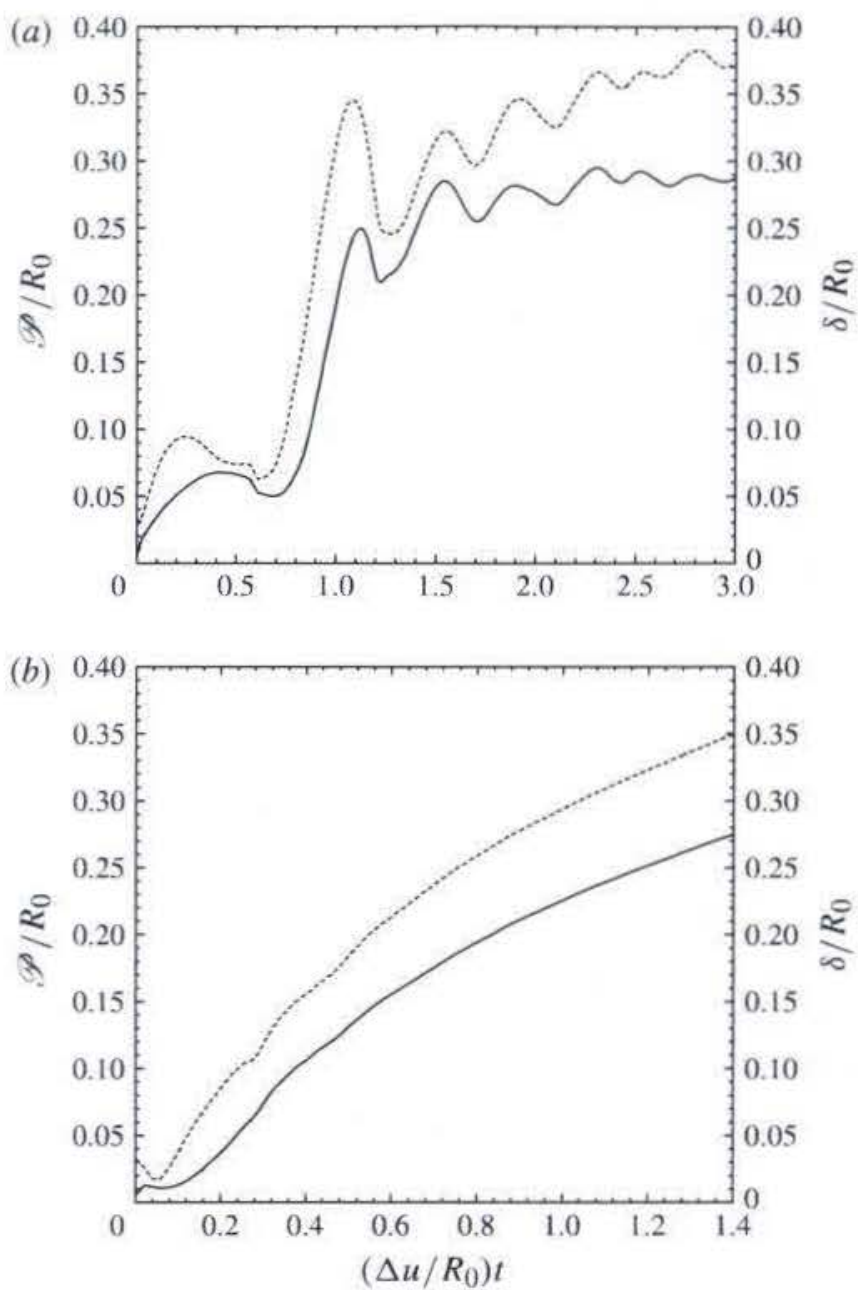

FIGURE 1. Total molecular mixing $\mathscr{P}$ (solid line) and maximum molecular mixing $\delta$ (dashed) versus $t:(a)$ air $\rightarrow \mathrm{SF}_{6}$ and $(b) \mathrm{SF}_{6} \rightarrow$ air.

normalized turbulent mass flux $a_{i}$ in the $i$ th direction and the Favre Reynolds stress components $R_{i j}$, especially the TKE $\mathscr{K}$, defined respectively as

$$
a_{i}=\frac{\left\langle\rho^{\prime} u_{i}^{\prime}\right\rangle}{\langle\rho\rangle}, \quad R_{i j}=\left\langle\rho u_{i}^{\prime \prime} u_{j}^{\prime \prime}\right\rangle, \quad \mathscr{K}=\frac{R_{k k}}{2\langle\rho\rangle} .
$$

While the density variance $\left\langle\rho^{2}\right\rangle$ mediates the turbulent mass flux in Boussinesq flows, in variable-density mixing (Livescu \& Ristorcelli 2007) this role is played by the density-specific volume correlation, or density self-correlation,

$$
b=-\left\langle\rho^{\prime} v^{\prime}\right\rangle, \quad \text { with } v=\frac{1}{\rho} .
$$

The quantity $b$ affects the production of radial turbulent mass flux $a$, through the term $b \partial\langle p\rangle / \partial r$ (see appendix A.2), and $a_{r}$ sets the energy conversion rate through the production term $a_{r} \partial\langle p\rangle / \partial r$ in the surface-averaged TKE equation (see appendix A.3).

Figure 3 depicts the radial profile of $b$ at three different late times, $t=2 t_{\text {Res } 1}, 3 t_{\text {Res }}$ and $4 t_{\text {Res } 1}$. The bimodal character of the light-heavy $b$-profile (figure $3 a$ ) persists as the spikes and bubbles continue to transport partially mixed gases from the outer 

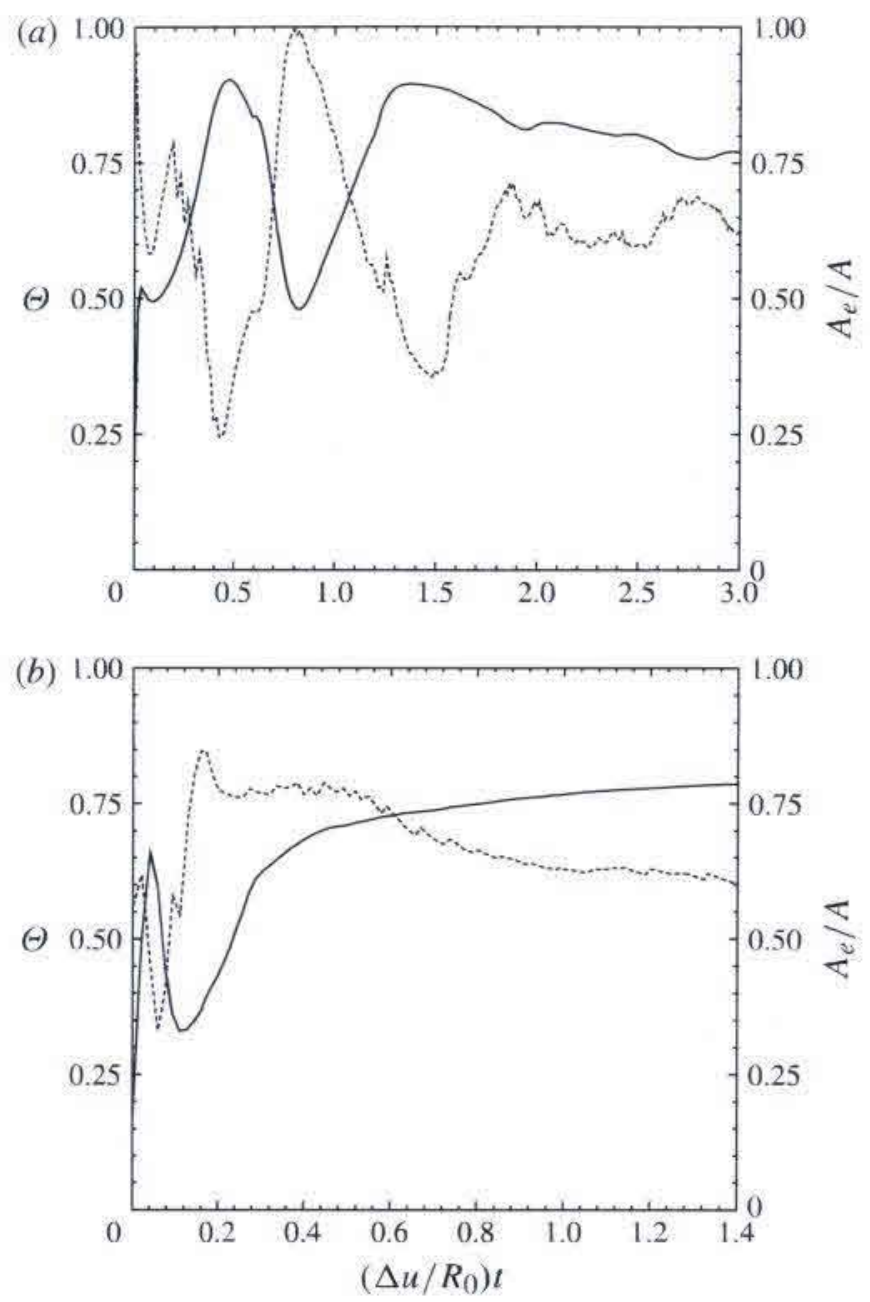

FIGURE 2. Ratio of mixed to entrained fluid $\Theta$ (solid line) and effective Atwood number ratio $A_{e} / A$ (dashed) versus $t:(a)$ air $\rightarrow \mathrm{SF}_{6}$ and $(b) \mathrm{SF}_{6} \rightarrow$ air.

regions of the mixing layer. As the mixing zone grows, it becomes more asymmetric, with a stronger peak on the heavy side, as confirmed by a positive peak of the $\partial b / \partial t$ profile on the heavy side (not shown here). The heavy-light $b$-profile evolves more into a single peak almost aligned with the layer centre as observed in planar RT mixing (Livescu et al. 2009), but still with a slight asymmetry towards the heavy side.

The study of $b$ encompasses the previous analysis of $A_{e}$ that was a measure of the density fluctuations at the layer centre: the Boussinesq approximation can be seen as the leading order of the Taylor expansion of $b$ for small density fluctuations $\epsilon_{\rho}=$ $\left\langle\rho^{\prime 2}\right\rangle^{1 / 2} /\langle\rho\rangle \ll 1$ (the value $\epsilon_{\rho}=0.05$ is usually taken to define the limit below which the Boussinesq approximation applies):

$$
b=\frac{\left\langle\rho^{\prime 2}\right\rangle}{\langle\rho\rangle^{2}}\left[1-\epsilon_{\rho} \frac{\left\langle\rho^{\prime 3}\right\rangle}{\left\langle\rho^{\prime 2}\right\rangle^{3 / 2}}+\epsilon_{\rho}^{2} \frac{\left\langle\rho^{\prime 4}\right\rangle}{\left\langle\rho^{2}\right\rangle^{2}}-\epsilon_{\rho}^{3} \frac{\left\langle\rho^{\prime 5}\right\rangle}{\left\langle\rho^{\prime 2}\right\rangle^{5 / 2}}+\cdots\right],
$$

where the odd terms characterize the skewness of the density profile. Differences between $b$ and the normalized density variance $\epsilon_{\rho}^{2}$ indicate non-Boussinesq effects already anticipated by the asymmetric growth of the mixing layer. The departure 
(a)
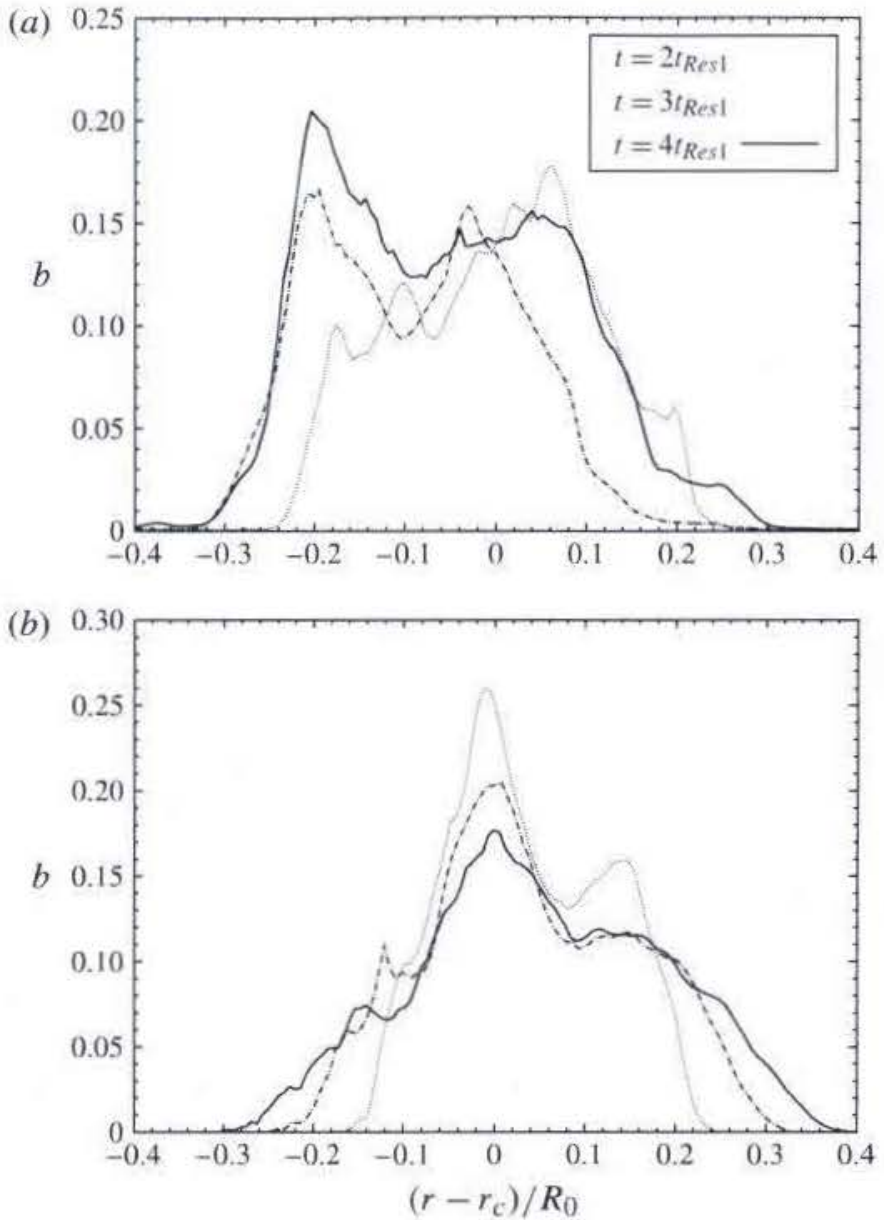

FIGURE 3. Radial $b$-profile (in the frame of the moving layer) at three different late times: (a) air $\rightarrow \mathrm{SF}_{6}$ and $(b) \mathrm{SF}_{6} \rightarrow$ air.

from the Boussinesq approximation is largest at the edges of the layer, consistent with figure 4 . Indeed, even though $\epsilon_{\rho}$ is small towards the edges (typically $<0.1$ at the edges, and $>0.3$ at the interior), the skewness of the density profile is positive on the light side and negative on the heavy side, all odd terms in (2.8) thus being of the same sign away from the layer centre. We further observe from figure 4 that $b$ has larger magnitude than $\epsilon_{\rho}^{2}$ on the heavy side, and smaller magnitude on the light side. Therefore, at fixed Atwood ratio, the Boussinesq equations would lead to smaller energy conversion rate on the heavy side of the layer, compared with the more general variable-density equations.

The surface-averaged equation governing the evolution of $b(r, t)$ is given by (see appendix A.1)

$$
\begin{aligned}
\frac{\partial b}{\partial t}+\tilde{u}_{r} \frac{\partial b}{\partial r}= & \underbrace{2 a_{r} \frac{\partial b}{\partial r}}_{(b \mathrm{I})} \underbrace{2 \frac{(1+b)}{\langle\rho\rangle} a_{r} \frac{\partial\langle\rho\rangle}{\partial r}}_{(b \mathrm{II})}+\underbrace{\frac{\langle\rho\rangle}{r^{2}} \frac{\partial}{\partial r}\left(r^{2} \frac{\left\langle\rho^{\prime} v^{\prime} u_{r}^{\prime}\right\rangle}{\langle\rho\rangle}\right)}_{(b \mathrm{III})} \\
& +\underbrace{2\langle\rho\rangle\left[\langle\boldsymbol{\nabla} \cdot \boldsymbol{u}\rangle-\frac{\langle v\rangle}{r^{2}} \frac{\partial}{\partial r}\left(r^{2}\left\langle u_{r}\right\rangle\right)\right]}_{(b \mathrm{IV})} .
\end{aligned}
$$



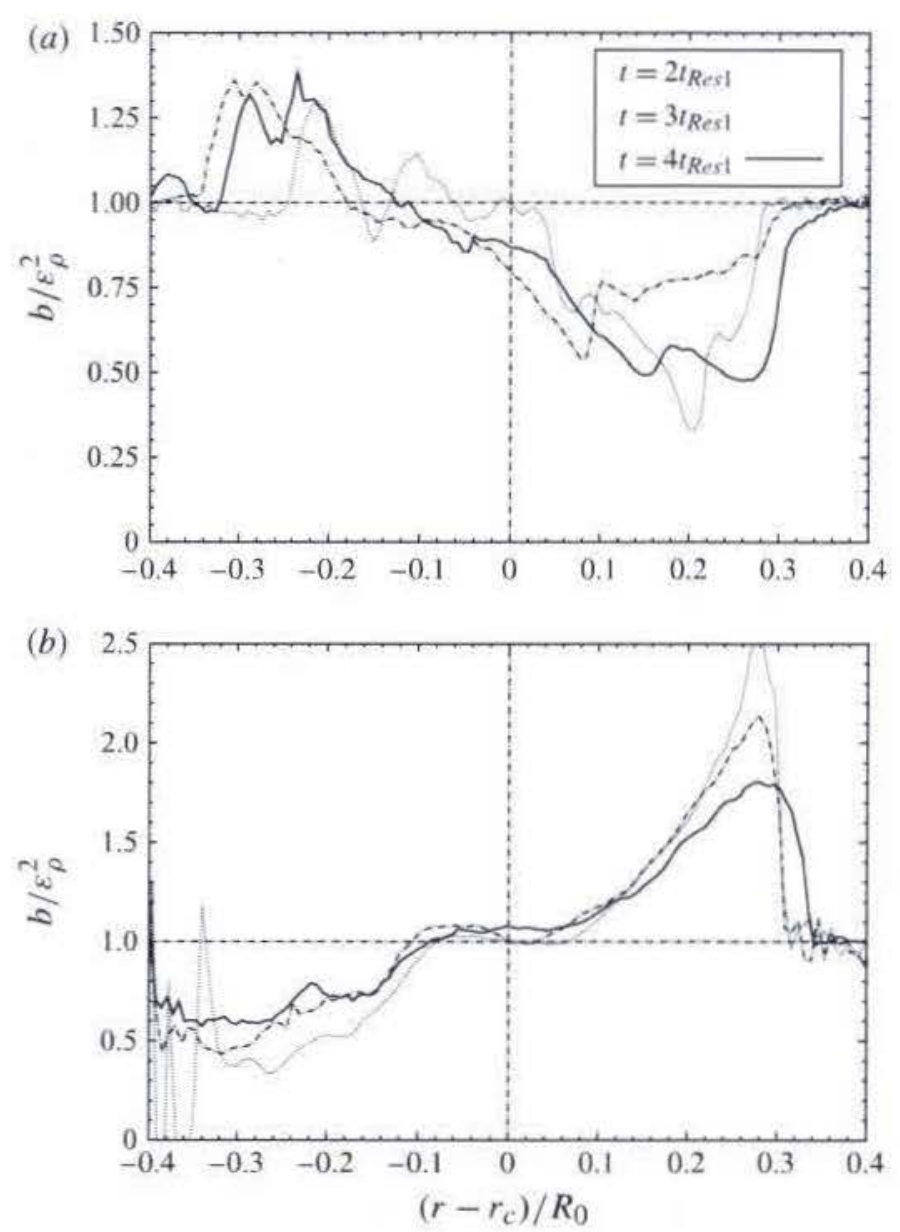

FIGURE 4. Ratio of $b$ to $\epsilon_{p}^{2}$ across the layer, at three different late times: $(a)$ air $\rightarrow \mathrm{SF}_{6}$ and $(b) \mathrm{SF}_{6} \rightarrow$ air.

Studying its right-hand side, as well as those of the $a_{i}$ and $\mathscr{K}$ equations (see appendices A.2 and A.3), is relevant to the characterization of the turbulent mixing and to second-order moment Reynolds-averaged Navier-Stokes (RANS) modelling in variable-density flows. Figure 5 measures the relative norm of each term (across the mixing layer) as a function of time, depicting that term ( $b$ IV), i.e. the production of $b$ by specific volume-dilatation correlations, is the dominant term at late times. In the light-heavy case, ( $b$ IV) actually accounts for more than $80 \%$ of the right-hand side across the whole layer, the other terms cancelling each other out due to opposite signs. The late-time approximate $b$-equation is therefore

$$
\frac{\partial b}{\partial t} \simeq 2\langle\rho\rangle\left[\langle v \nabla \cdot \boldsymbol{u}\rangle-\frac{\langle v\rangle}{r^{2}} \frac{\partial}{\partial r}\left(r^{2}\left\langle u_{r}\right\rangle\right)\right] .
$$

In the heavy-light case, term ( $b$ IV) is still dominant. When accounting for possible cancellation of terms with opposite signs, we obtain the same equation as (2.1.0) near the edges of the layer. As noted by Livescu et al. (2009) for planar RT mixing, we find presently that $(b$ II) almost balances $(b$ IV) at the layer interior, as they have close magnitude but opposite sign:

$$
0 \simeq-\frac{(1+b)}{\langle\rho\rangle} a_{r} \frac{\partial\langle\rho\rangle}{\partial r}+\langle\rho\rangle\left[\langle v \nabla \cdot \boldsymbol{u}\rangle-\frac{\langle v\rangle}{r^{2}} \frac{\partial}{\partial r}\left(r^{2}\left\langle u_{r}\right\rangle\right)\right] .
$$



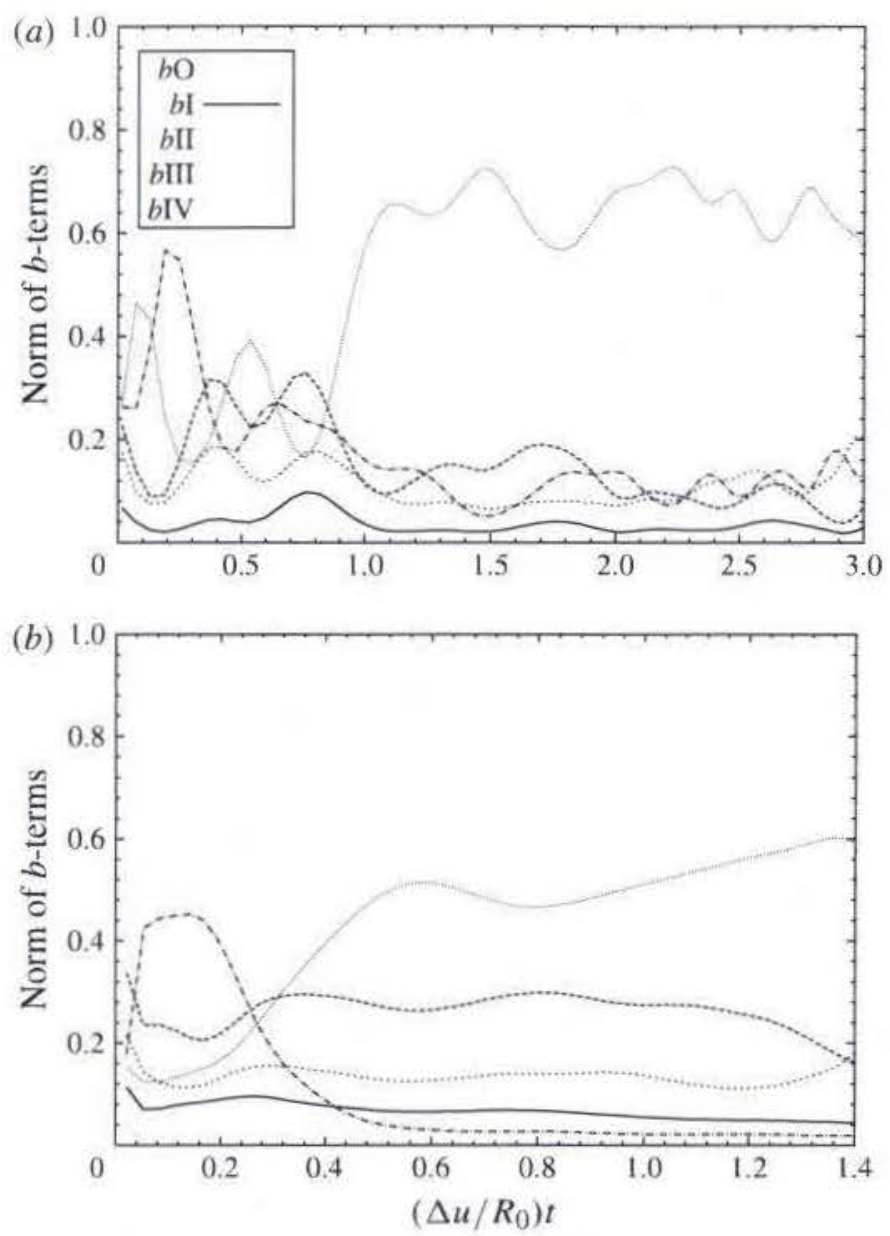

FIGURE 5. Relative norm of the right-hand side terms of the $b$-equation versus $t:(a)$ air $\rightarrow \mathrm{SF}_{6}$ and $(b) \mathrm{SF}_{6} \rightarrow$ air.

Hence, a model for the production of $b$ by the mean density gradient, $(b$ II), would also be necessary for RANS closure in the heavy-light configuration.

\section{TKE and dissipation}

\subsection{Subgrid and resolved TKE and turbulent dissipation rate}

We compute two kinds of surface-averaged statistics of the turbulent activity: (i) the total TKE (per unit mass) defined as the sum of the resolved-scale TKE, $\mathscr{K}_{\text {res }}$, and the SGS TKE, $\mathscr{K}_{\text {sgs }}$, and (ii) the total turbulent dissipation rate as the sum of the resolved-scale dissipation, $\varepsilon_{\text {res }}$, and the subgrid energy transfer, $\varepsilon_{s g s}$, that are given for example in Lombardini (2008):

$$
\begin{gathered}
\mathscr{K}_{\text {res }}=\frac{1}{2} \widetilde{u_{i}^{\prime \prime} u_{i}^{\prime \prime},} \quad \mathscr{K}_{\text {sgs }}=\frac{\left\langle\tau_{i i}\right\rangle}{2\langle\rho\rangle} . \\
\varepsilon_{\text {res }}=\frac{\left\langle d_{i j}^{\prime} S_{i j}^{\prime}\right\rangle}{\langle\rho\rangle}, \quad \varepsilon_{s g s}=-\frac{\left\langle\tau_{i j}^{\prime} S_{i j}^{\prime}\right\rangle}{\langle\rho\rangle} .
\end{gathered}
$$

Here $d_{i j}$ and $-\tau_{i j}$ are the components of the resolved and subgrid stress tensors, the total stress tensor being $\sigma_{i j}=d_{i j}-\tau_{i j} ; S_{i j}$ denote the components of the resolved-scale 

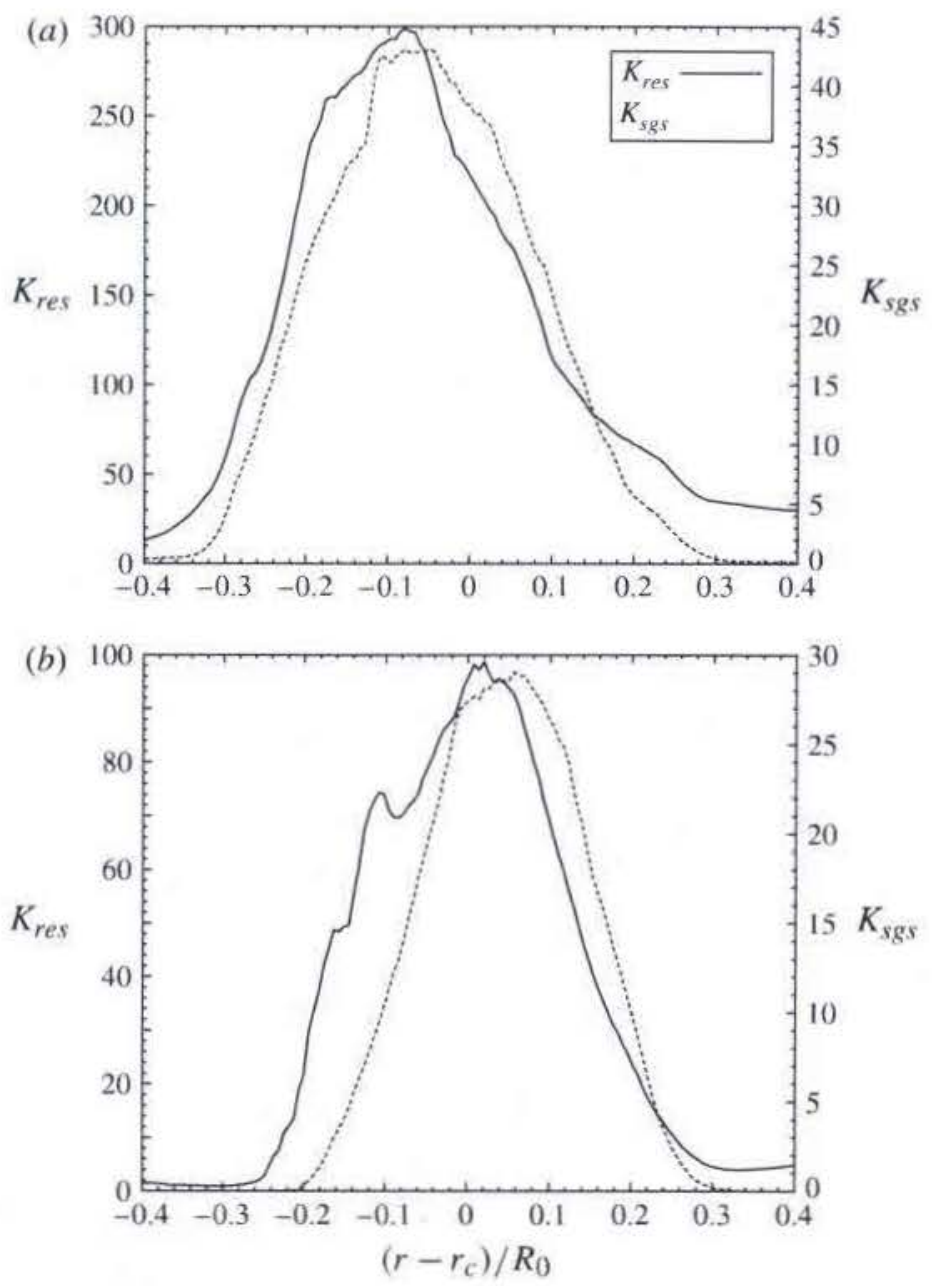

FIGURE 6. Radial profile of the resolved (solid line) and subgrid (dashed line) TKE at the late time $t=3 t_{\text {Res } 1}:(a)$ air $\rightarrow \mathrm{SF}_{6}$ and $(b) \mathrm{SF}_{6} \rightarrow$ air.

strain-rate tensor. Typical surface-averaged profiles of the resolved and subgrid TKE are shown in figure 6, at late times. Surface averages of the resolved and subgrid energy dissipation rates are plotted in figure 7. In particular, the subgrid energy transfer off the grid is much larger than the (resolved) viscous dissipation (by a factor of approximately 1000 for air $\rightarrow \mathrm{SF}_{6}$ and 500 for $\mathrm{SF}_{6} \rightarrow$ air), implying that most of the dissipation is provided by the subgrid activity as commonly observed in LES of turbulent flows. Even at three times the reshock time, the turbulent activity is still intense. The total TKE and turbulent dissipation seem to peak on the heavy (bubble) side, at $\langle Y\rangle \approx 0.5$, confirming some of the observations on the mixing-layer asymmetry made in $\$ 2.2$.

The evolution of the volume-averaged total TKE, $\mathscr{K}^{v o l}$, computed by summing radially across the mixing layer the total surface-averaged TKE, $\mathscr{K}_{\text {res }}+\mathscr{K}_{\text {sgs }}$, is displayed in figure 8. In the light-heavy configuration (figure $8 a$ ), the large deposition of baroclinic energy due to the first reshock and the RT-unstable period following (figure $3 a$ of Part 1) manifests itself by a large rise in $\mathscr{K}^{v o l}$, for $\Delta u t / R_{0} \gtrsim 0.6$. After these events $\left(\Delta u t / R_{0} \gtrsim 0.8\right)$, the TKE decays until a second reshock/RT-unstable period deposits additional vorticity. Similar events occur at later times with weaker signatures. To compare, in the planar case where (i) the first reshock is followed by 

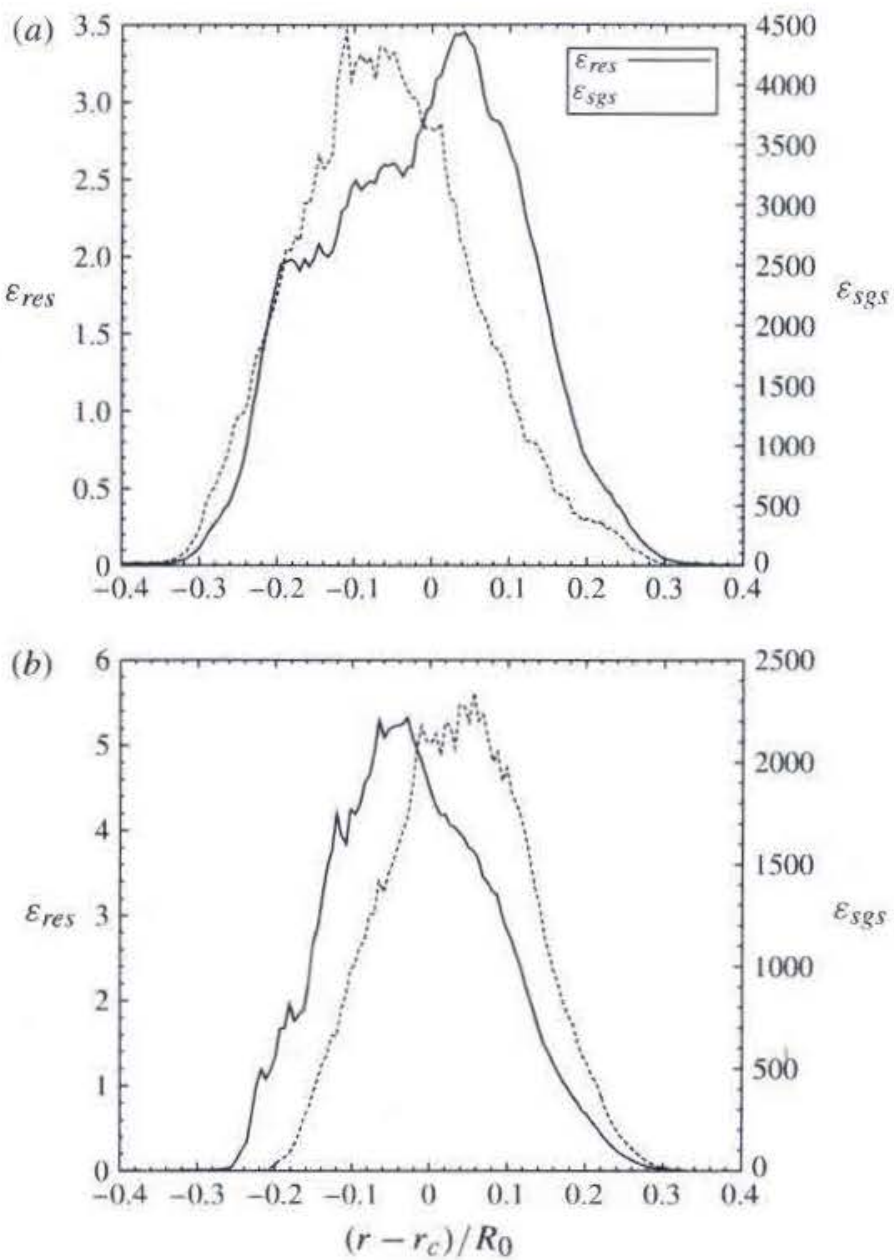

FIGURE 7. Radial profile of the resolved (solid line) and subgrid (dashed line) turbulent dissipation rates at the late time $t=3 t_{\text {Res } 1}:(a)$ air $\rightarrow \mathrm{SF}_{6}$ and $(b) \mathrm{SF}_{6} \rightarrow$ air.

a sequence of expansion fans reverberating between the endwall and the mixing layer, and (ii) there is no occurrence of RT instability, the TKE experiences a slow but persistent decay following the first reshock- and expansion-contact interaction, and none of the secondary expansion fans generate a sufficiently large pressure gradient to deposit noticeable amounts of vorticity. In the heavy-light configuration (figure $8 b$ ), the first reshock interaction leads to an increase in $\mathscr{K}^{v o l}$, for $\Delta u t / R_{0} \gtrsim 0.27$. After this event follows a period of decay until a second reshock deposits additional vorticity. Subsequent reshocks of weaker intensity are almost imperceptible.

\subsection{Turbulent length scales}

To investigate the nature of the decaying turbulence observed in the previous subsection, we define various turbulent scales. First, an integral length $\ell_{\varepsilon}$ to be assimilated to a dissipation length as classically defined in isotropic turbulence, is specified as follows:

$$
\ell_{\varepsilon}=\frac{\mathrm{u}^{\prime 3}}{\varepsilon}, \quad \text { with } \mathrm{u}^{\prime}=\sqrt{\frac{2\left(\mathscr{K}_{\text {res }}+\mathscr{K}_{\text {sgs }}\right)}{3}} \text { and } \varepsilon=\varepsilon_{\text {res }}+\varepsilon_{\text {sgs }} \text {. }
$$



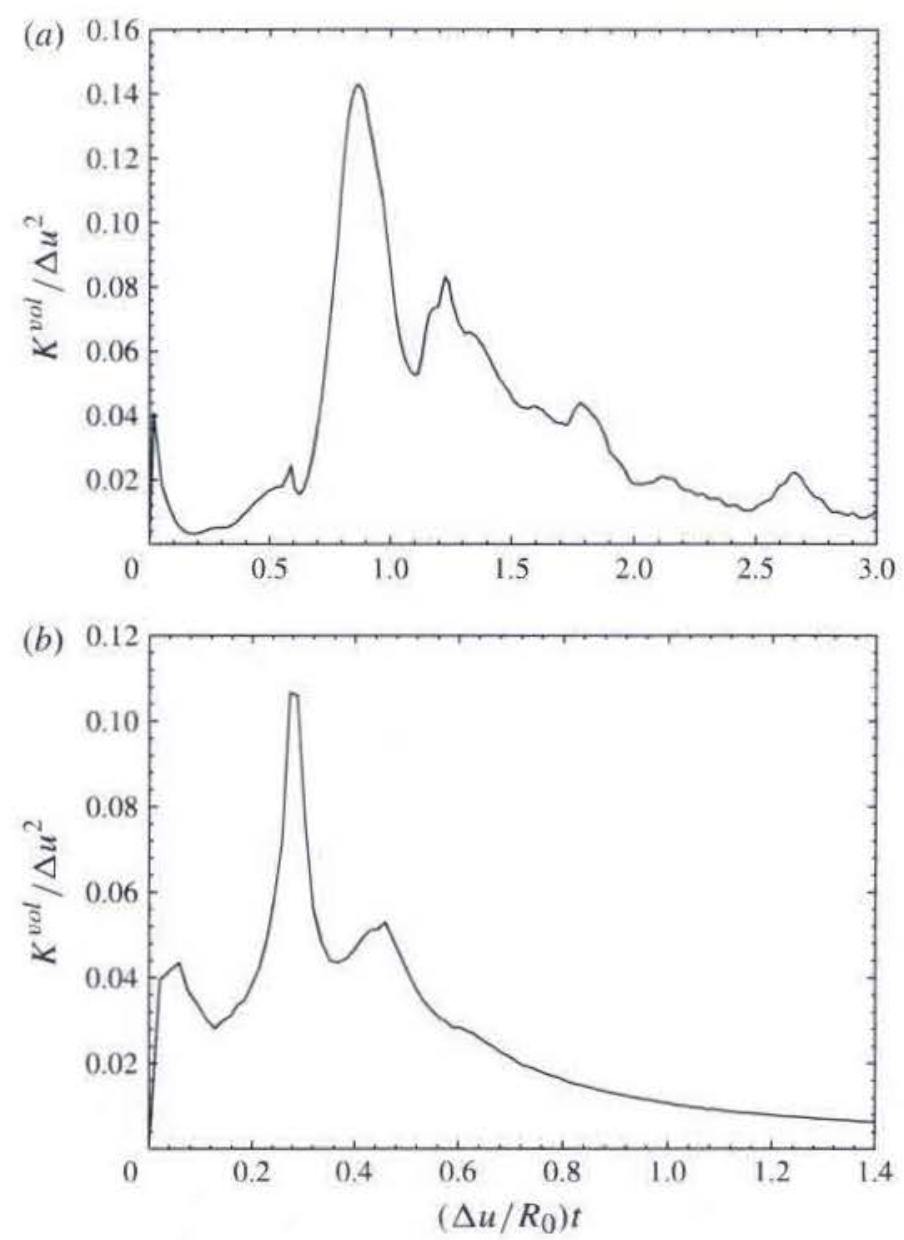

FIGURE 8. Evolution of the volume-averaged total TKE: $(a)$ air $\rightarrow \mathrm{SF}_{6}$ and $(b) \mathrm{SF}_{6} \rightarrow$ air.

A Taylor microscale is also computed with the following isotropic definition,

$$
\lambda_{T}=\frac{\mathrm{u}^{\prime}}{\sqrt{\varepsilon /(15\langle v\rangle)}},
$$

where $\langle v\rangle$ is the surface-averaged kinematic viscosity of the mixture. Taylor-like microscales can also be derived for fields other than the velocity. For example, for the scalar field,

$$
\lambda_{Y}=\sqrt{\frac{\left\langle Y^{\prime 2}\right\rangle}{\left\langle\nabla Y^{\prime} \cdot \nabla Y^{\prime}\right\rangle / 3}} .
$$

The definition (3.4) results from a simple analogy with the TKE dissipation: the dissipation rate ( $\mathscr{K}$ VI) in the surface-averaged TKE transport equation (A 5), i.e. the sum of $(3.1 \mathrm{c})$ and $(3.1 \mathrm{~d})$, is consistent with the definition of the Taylor microscale (3.3). Here, the scalar dissipation rate $\chi_{s}=\langle D\rangle\left\langle\nabla Y^{\prime} \cdot \nabla Y^{\prime}\right\rangle$, which arises in the surface-averaged transport equation for the scalar variance $\left\langle Y^{2}\right\rangle / 2$, results in a Taylor-like scalar microscale $\lambda_{Y}^{2} \sim\left\langle Y^{\prime 2}\right\rangle /\left(\chi_{s} /\langle D\rangle\right)$, with $\langle D\rangle$ the surface-averaged mass diffusion coefficient of the mixture. A factor of three is added to obtain a proper isotropic definition. 
We plot in figure 9 the ratio of the time scales associated with the energy and the scalar dissipations,

$$
r_{d}(t)=\frac{3 \mathrm{u}^{\prime 2} / \varepsilon}{\left\langle Y^{\prime 2}\right\rangle / \chi_{s}}=\frac{3}{5} \frac{1}{S_{c}} \frac{\lambda_{T}^{2}}{\lambda_{Y}^{2}},
$$

where $S_{c}=\langle v\rangle /\langle D\rangle$ and the various surface averages are evaluated the mixing-layer centre. The time-scale ratio has often been employed to characterize the mixing in forced turbulence in a constant mean gradient of density or temperature. It represents the non-dimensional mix rate in time units scaled by $\mathrm{u}^{2 /} / \varepsilon$ (Ristorcelli 2006). Figure 9, in particular figure $9(b)$, shows a different behaviour than in Boussinesq mixing for which the rate is assumed constant (Warhaft \& Lumley 1978). In variable-density mixing, the ratio can vary considerably, specially at high Atwood ratios and Reynolds numbers. However, Livescu \& Ristorcelli (2008) indicate that, in the decay stage of their variable-density buoyancy-driven turbulent mixing test, the ratio becomes Boussinesq-like and reaches a value near the isotropic turbulence value of 2 (in the case $S c=1$ ). Pullin (2000) observes, with the same subgrid model as the present one, values of the ratio $\approx 2.8$ over a range of increasing Taylor-scale Reynolds numbers $>200$, for a test consisting of passive scalar transport by forced isotropic turbulence with a preserved mean scalar gradient. For similar flows, the direct numerical simulation of Overholt \& Pope (1996) and Gotoh, Watanabe \& Suzuki (2011) at Taylor-scale Reynolds numbers $<200$ and 600 , respectively, and the implicit LES of Wachtor et al. (2013) at Taylor-scale Reynolds numbers $<250$, show continued growth of $r_{d}$ over the range of increasing Reynolds numbers simulated. Here we measured, at the mixing-layer centre and at late times in the evolution of the decaying turbulent mixing, values of the Taylor-scale Reynolds numbers $\gtrsim 1000$ (not shown). It is not clear why, at such large Reynolds numbers, our LES results show larger values of $r_{d}$ in the heavy-light case than in the light-heavy one, but this could be a useful interrogation when assessing mixing rate closures. Moreover, a study of the Reynolds number dependence of $r_{d}$ in the late-time decaying turbulent mixing stages would require the investigation of different incident Mach numbers.

The length scale (3.4) represent the characteristic wavelength of a perturbation field, more precisely a measure of the root mean square slope of the perturbed interface. Mathematically, they are also referred to as the zero-crossing wavelength, which is an indicator of the spectral frequency modes present within the interface.

Finally, the Kolmogorov scale $\lambda_{K}$, characterizing the very smallest, dissipative eddies, is given by

$$
\lambda_{K}=\left(\frac{\langle v\rangle^{3}}{\varepsilon}\right)^{1 / 4} .
$$

All of these scales are evaluated at the mixing-layer centre and their evolution is represented in figure 10 . While the early growth of the mixing layer is expected to be mainly anisotropic, with a strong radial signature at all scales, we might expect a trend to isotropy at later times, once the main reshock waves have exited the computational domain. Therefore, we do not attach significance to the above definitions when $\Delta u t / R_{0} \lesssim 1$ in the light-heavy case and $\lesssim 0.5$ for the heavy-light case, but we adopt them for a first description of the decaying turbulence phase of the flow. At such late times, a large separation of scales is observed between the integral length scale (3.2) and the Kolmogorov scale (3.6). Note that, for both light-heavy and heavy-light configurations, the grid size is well within the inertial subrange, justifying a posteriori the need of a subgrid model. 

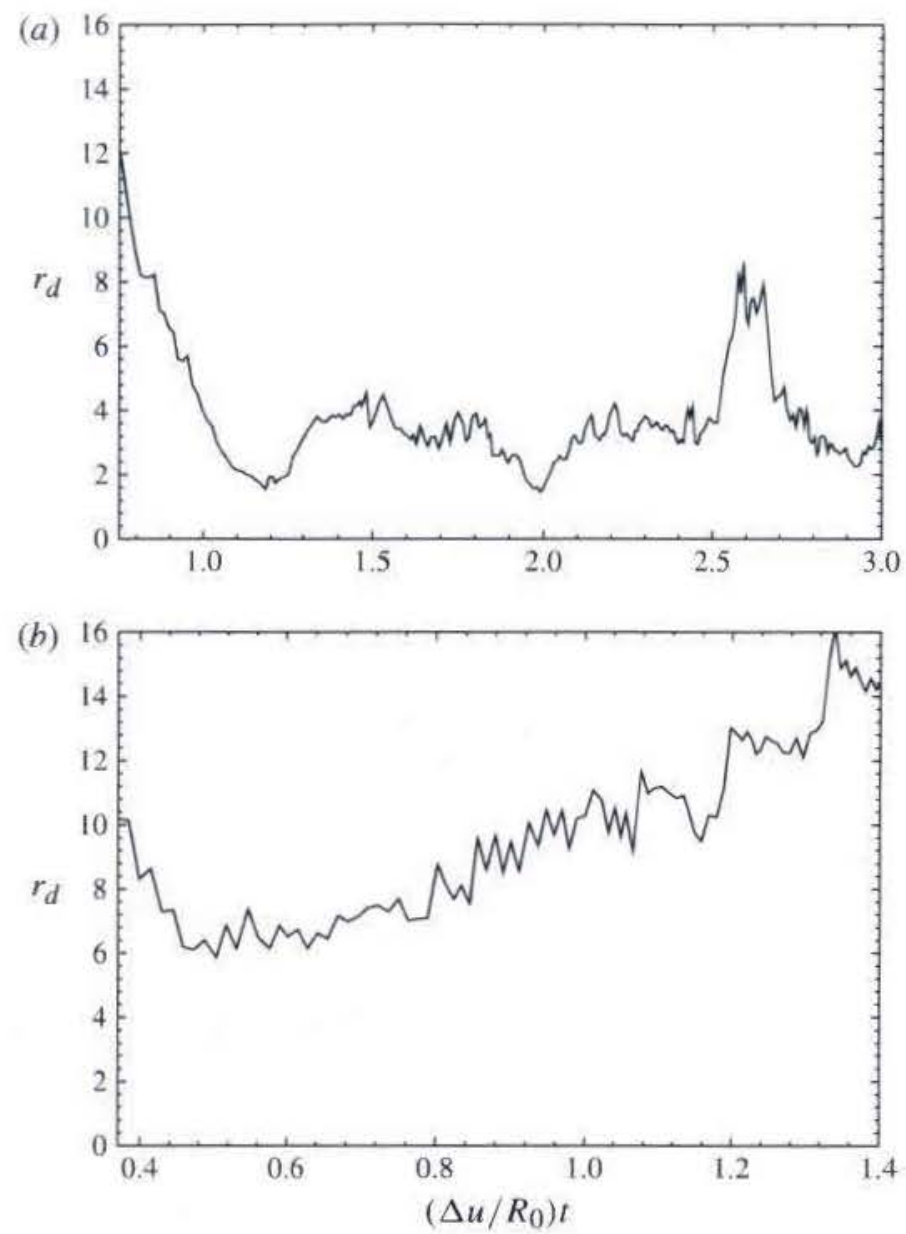

FIGURE 9. Evolution of the time ratio $r_{d}(t):(a)$ air $\rightarrow \mathrm{SF}_{6}$ and $(b) \mathrm{SF}_{6} \rightarrow$ air.

\subsection{Anisotropy}

Since the driving flow is anisotropic, it is expected that the normal stresses will be anisotropic too. The large-scale anisotropy, which appears in the turbulent mass flux and TKE equations (appendix A), is measured by the Favre Reynolds stress anisotropy tensor

$$
B_{\alpha \beta}=\frac{R_{\alpha \beta}}{R_{k k}}-\frac{1}{3} \delta_{\alpha \beta}, \quad \alpha, \beta=r, \theta, \phi .
$$

A diagonal component reaches zero for isotropy, its upper bound, $2 / 3$, when there is $100 \%$ of the energy in that component, and its lower bound, $-1 / 3$, when there is no energy in that component. Similar to variable-density mixing in buoyancy-driven turbulence (Ristorcelli \& Clark 2004; Livescu \& Ristorcelli 2007), the normal stresses are non-isotropic at late times (figure 11). In the heavy-light case, $B_{r r}$ seems to evolve between the values 0.15 and 0.3 , corresponding to $\approx 50$ to $65 \%$ energy in the radial component. This is comparable to RT turbulence (Livescu et al. 2009) in which $B_{r r}$ evolves to an almost constant asymptotic value of $\approx 0.3$ across the layer. A slight asymmetry is apparent here, with a stronger anisotropy on the light side. The lightheavy configuration exhibits a significantly different structure, another signature of the successive RT-unstable events. After four times the reshock time, $B_{r r}$ does not seem to have reached an asymptotic state yet. At such times, the profile is asymmetric, with 
(a)

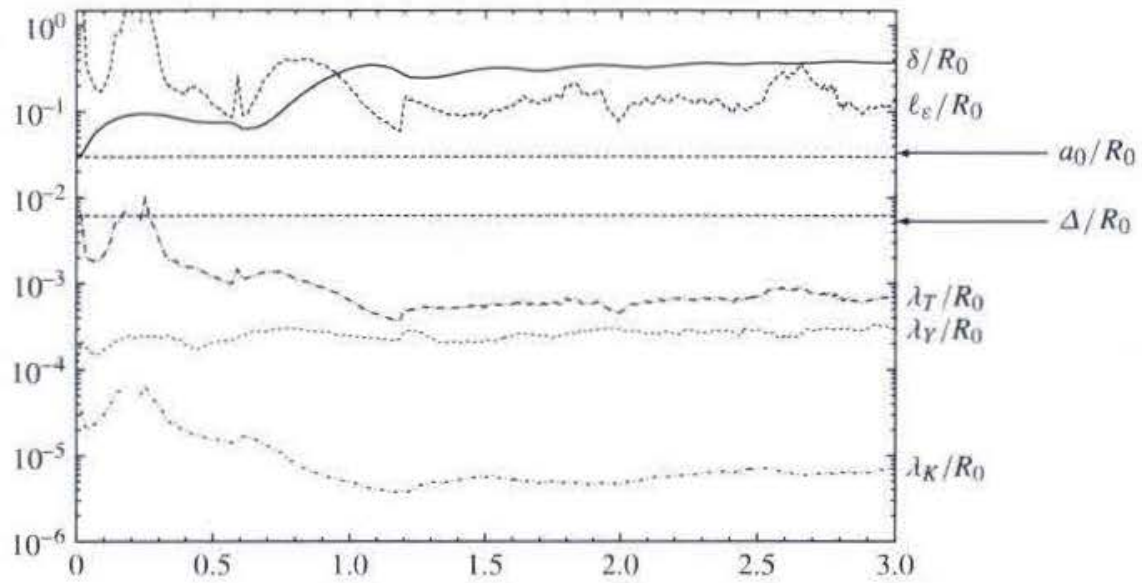

(b)

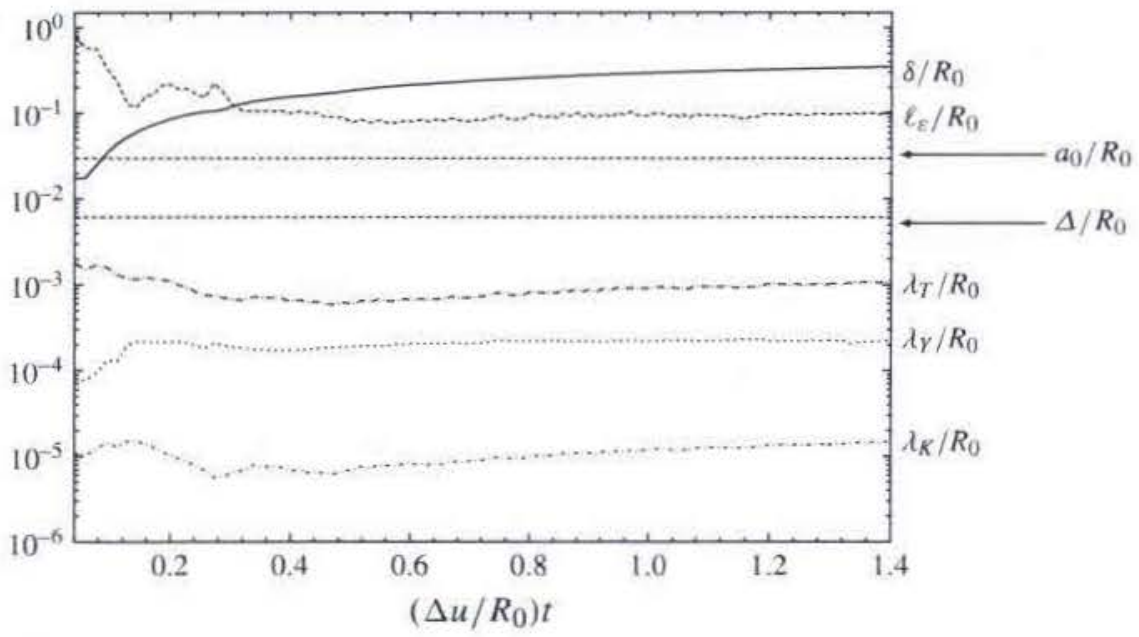

FIGURE 10. Evolution of various length scales evaluated at the mixing-layer centre $r=r_{c}$ : mixing-layer width $\delta$, integral length $\ell_{\varepsilon}$, scalar-based Taylor microscale $\lambda_{Y}$, Taylor microscale $\lambda_{T}$ and Kolmogorov microscale $\lambda_{K}$. (a) $\mathrm{Air} \rightarrow \mathrm{SF}_{6}$ and (b) $\mathrm{SF}_{6} \rightarrow$ air. The two thin, dashed, horizontal lines represent the finest grid size $\Delta$ (bottom line) and the initial perturbation amplitude $a_{0}$ (top line).

values in the $0.15-0.3$ range on the light side, and negative values on the heavy side corresponding to less than $30 \%$ energy in the radial component.

To account for the anisotropy of the flow, directional Taylor-scale Reynolds numbers are also introduced:

$$
\begin{gathered}
\operatorname{Re}_{\lambda_{T_{\alpha}}}=\frac{\mathrm{u}_{\alpha}^{\prime} \lambda_{T_{\alpha}}}{\langle v\rangle}, \quad \text { with } \lambda_{T_{\alpha}}=\frac{\mathrm{u}_{\alpha}^{\prime}}{\sqrt{\varepsilon_{\alpha} /(15\langle v\rangle)}}, \\
\mathrm{u}_{\alpha}^{\prime}=\sqrt{\widetilde{u_{\alpha}^{\prime \prime} u_{\alpha}^{\prime \prime}+\frac{\left\langle\tau_{\alpha \alpha}\right\rangle}{\langle\rho\rangle}}}, \quad \varepsilon_{\alpha}=15 \frac{\left\langle d_{\alpha \alpha}^{\prime} S_{\alpha \alpha}^{\prime}\right\rangle-\left\langle\tau_{\alpha \alpha}^{\prime} S_{\alpha \alpha}^{\prime}\right\rangle}{\langle\rho\rangle}, \quad \alpha=r, \theta, \phi .
\end{gathered}
$$

In these expressions, the directional TKE and dissipation rate are both the sum of a resolved and subgrid contribution, similarly to $(3, \mid a, b)$ and $(3,1 c, d)$. Expecting statistical isotropy in the transverse directions, we define a transverse Taylorscale Reynolds number by $R e_{\lambda T_{\theta \phi}}=\left(R e_{\lambda T_{\theta}}+R e_{\lambda T_{\phi}}\right) / 2$. We also define directional 

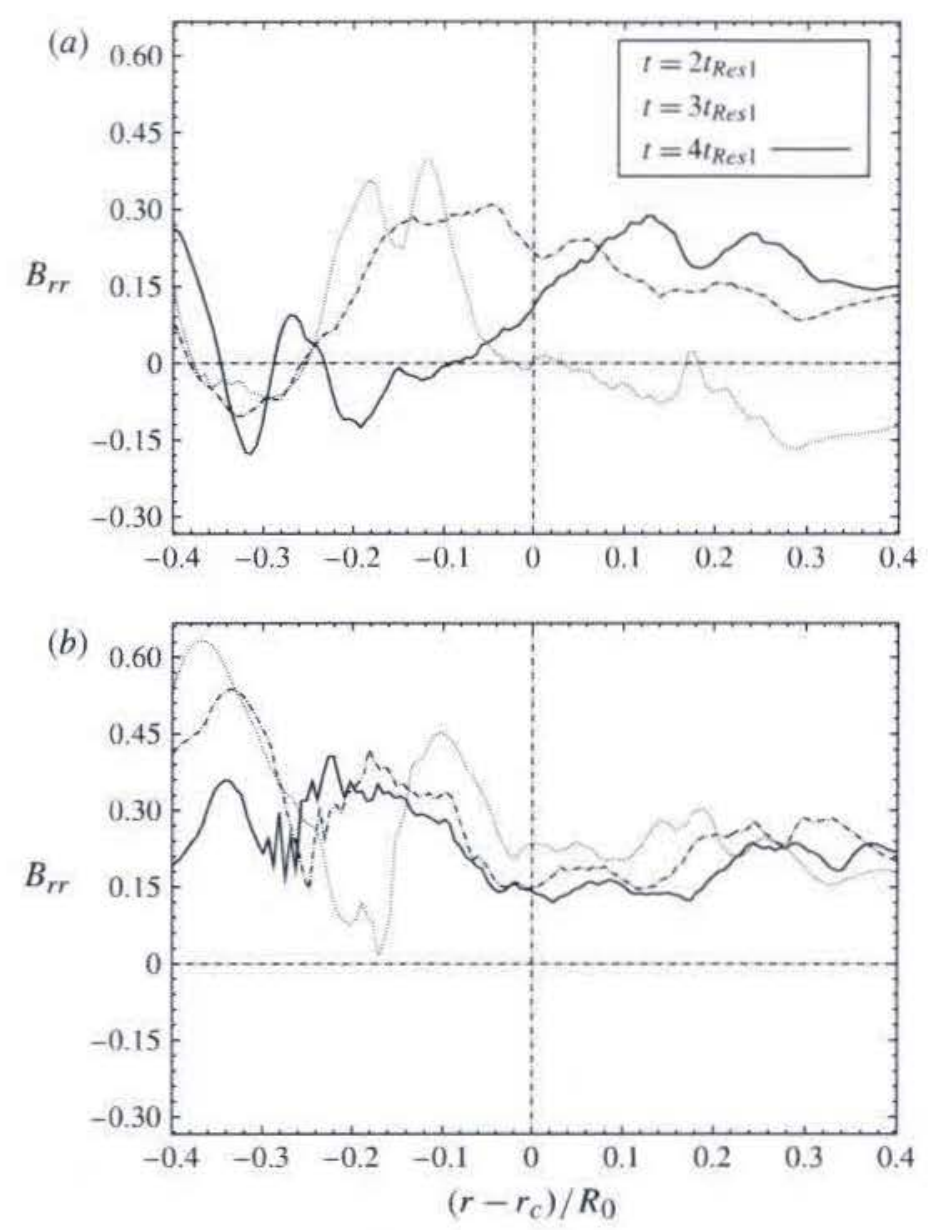

FIGURE 11. Radial profile of $B_{r}$, diagonal component of the Favre Reynolds stress anisotropy tensor, at three different late times: $(a)$ air $\rightarrow \mathrm{SF}_{6}$ and $\left(\right.$ b) $\mathrm{SF}_{6} \rightarrow$ air.

Kolmogorov microscales,

$$
\lambda_{K_{\alpha}}=\left(\frac{\langle v\rangle^{3}}{\varepsilon_{\alpha}}\right)^{1 / 4}, \quad \alpha=r, \theta, \phi .
$$

A transverse Kolmogorov microscale is then computed as $\lambda_{K_{\theta \phi}}=\left(\lambda_{K_{\theta}}+\lambda_{K_{\phi}}\right) / 2$. The Taylor and Kolmogorov directional measures are taken at the mixing-layer centre and their evolution is represented in figures 12 and 13 respectively. In both light-heavy and heavy-light configurations, isotropy at the Kolmogorov scales is practically reached at late times, while there is no evidence of isotropy at the Taylor microscales, suggesting anisotropy in the TKE components but not the turbulent dissipation. This speculation is confirmed by calculations of the variance of each velocity component at the mixing-layer centre, in particular by the results on $B_{r r}$ in the previous paragraph. We finally note in figure $12(a)$ the repeated RT-unstable periods typical of the light-heavy configuration.

\section{Spectral statistics on spherical surfaces}

Here we describe the spectral analysis of a statistically isotropic field on a spherical surface and, inversely, how to build a statistically isotropic field with a given spectral 

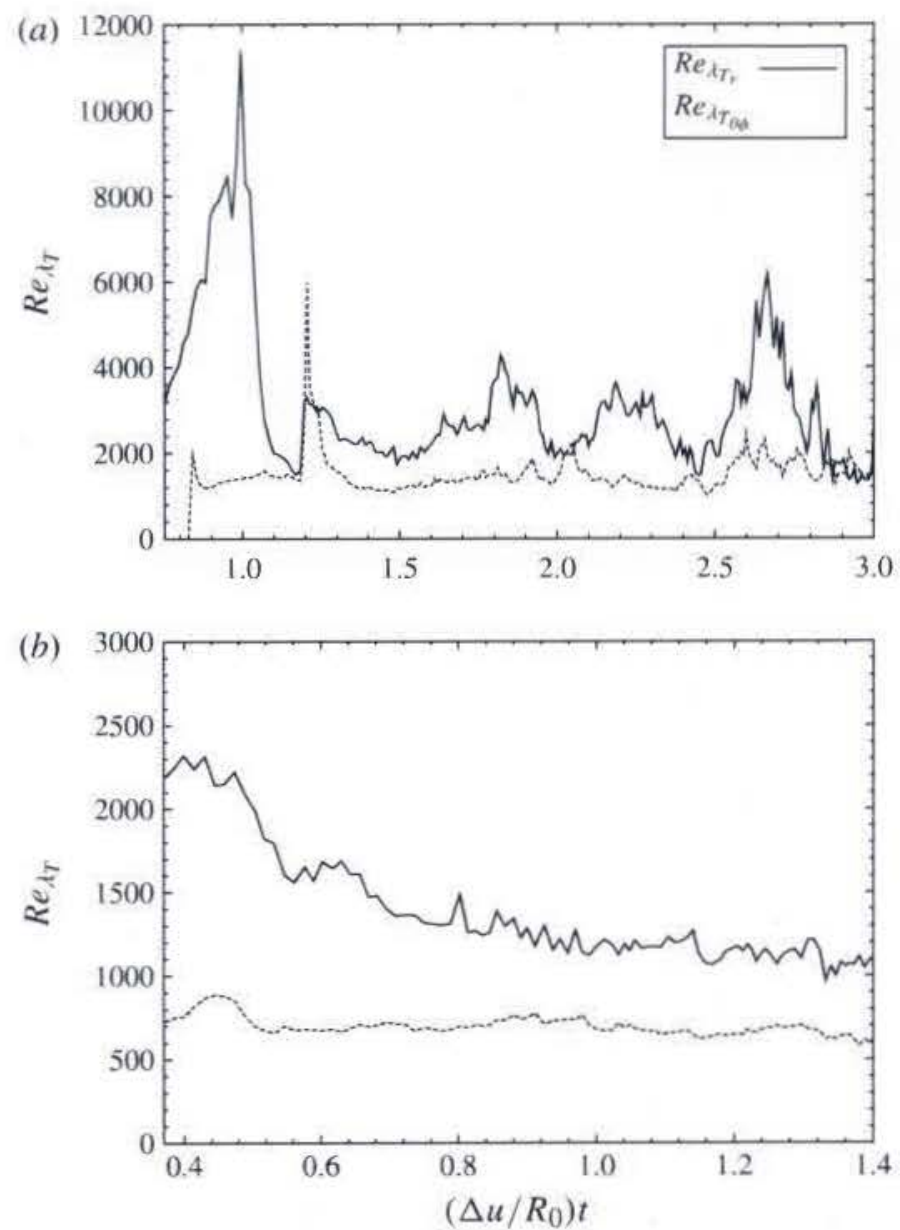

FIGURE 12. Late-time evolution of the radial (solid line) and transverse (dashed line) Taylor-scale Reynolds numbers, evaluated at $r=r_{c}:(a)$ air $\rightarrow \mathrm{SF}_{6}$ and $(b) \mathrm{SF}_{6} \rightarrow$ air.

signature. We then present some spectral results obtained from LES data of the spherical turbulent mixing.

\subsection{Decomposition using the spherical harmonics basis}

The spherical harmonics are the natural basis to represent a field on a sphere as they circumvent the pole singularity problem of a spherical coordinate system. On a sphere of radius $R$, any real square-integrable function can be expanded as a linear combination of the Laplace real spherical harmonics which form a complete set of orthonormal functions:

$$
f(R, \theta, \phi)=\sum_{\ell=0}^{\infty} \sum_{m=-\ell}^{\ell} \int_{\ell m} Y_{\ell m}(\theta, \phi),
$$

where the real spherical harmonics are given by:

$$
Y_{\ell m}(\theta, \phi)= \begin{cases}N_{(\ell . m)} P_{\ell}^{m}(\cos \theta) \cos (m \phi) & m \geqslant 0 . \\ N_{(\ell .|m|)} P_{\ell}^{|m|}(\cos \theta) \sin (|m| \phi) & m<0 .\end{cases}
$$



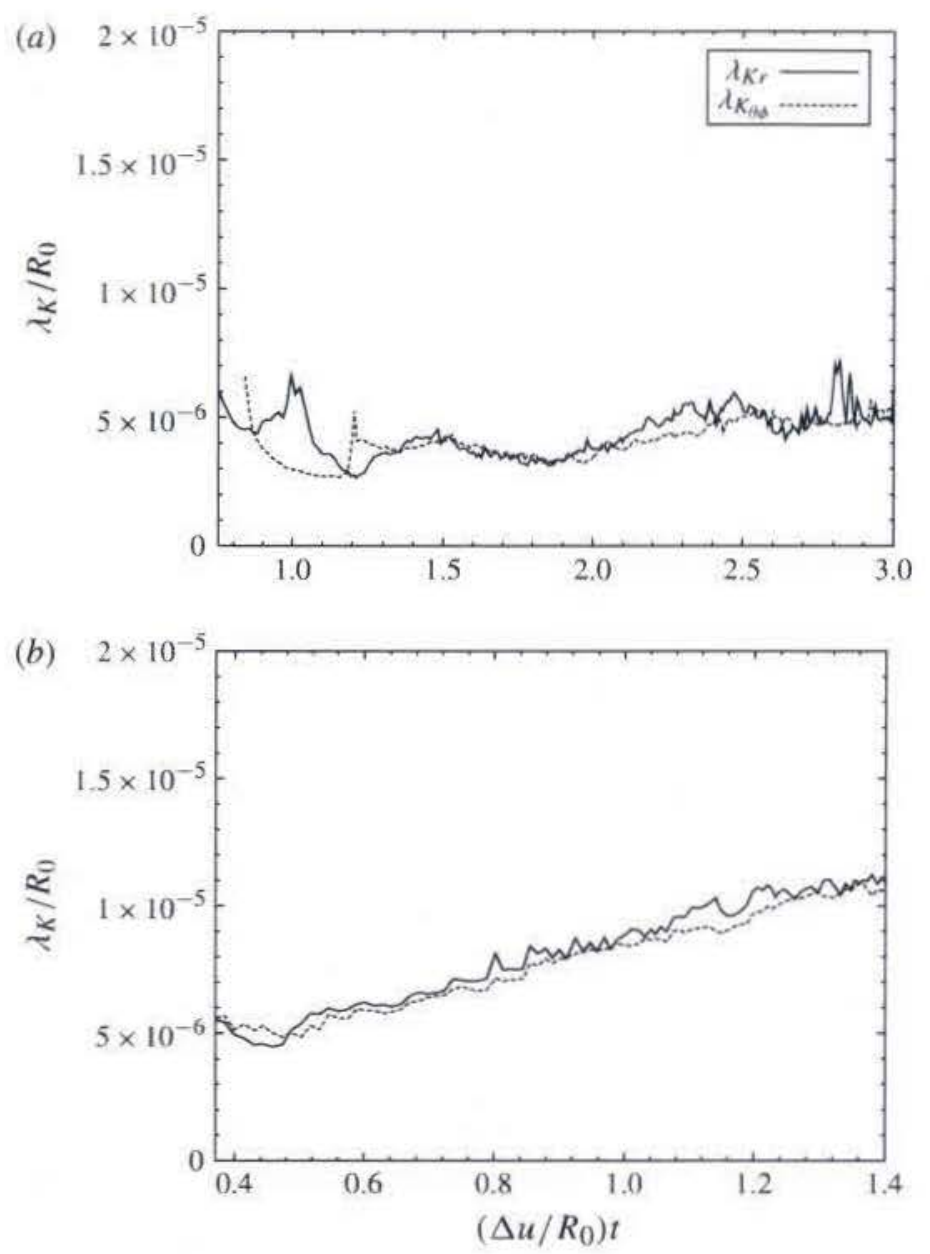

FIGURE 13. Late-time evolution of the radial (solid line) and transverse (dashed line) Kolmogorov microscales, evaluated at $r=r_{c}:(a)$ air $\rightarrow \mathrm{SF}_{6}$ and $(b) \mathrm{SF}_{6} \rightarrow$ air.

The $P_{\ell}^{m}$ are the associated Legendre polynomials and $N_{(\ell, m)}$ is a normalization constant given by

$$
N_{(\ell, m)}=\sqrt{\frac{\left(2-\delta_{0 m}\right)(2 \ell+1)(\ell-m) !}{(\ell+m) !}} .
$$

With this so-called 'unit-power' normalization,

$$
\frac{1}{4 \pi} \oiint_{\Omega} Y_{\ell m}(\theta, \phi) Y_{\ell^{\prime} m^{\prime}}(\theta, \phi) \mathrm{d} \Omega=\delta_{\ell \ell^{\prime}} \delta_{m m^{\prime}}
$$

From a given field $f(R, \theta, \phi)$, the coefficients $f_{\ell m}$ can therefore be computed as

$$
f_{\ell m}=\frac{1}{4 \pi} \oiint_{\Omega} f(R, \theta, \phi) Y_{\ell m}(\theta, \phi) \mathrm{d} \Omega .
$$




\subsection{Angular power spectrum}

Next we define the two-point correlation function of a random field $f$ on a sphere as

$$
\mathscr{C}=\overline{f\left(\boldsymbol{x}_{1}\right) f\left(\boldsymbol{x}_{2}\right)}=\sum_{\ell_{1}=0}^{\infty} \sum_{m_{1}=-\ell_{1}}^{\ell_{1}} \sum_{\ell_{2}=0}^{\infty} \sum_{m_{2}=-\ell_{2}}^{\ell_{2}} \overline{f_{\ell_{1} m_{1}} f_{\ell_{2} m_{2}}} Y_{\ell_{1} m_{1}}\left(\theta_{1}, \phi_{1}\right) Y_{\ell_{2} m_{2}}\left(\theta_{2}, \phi_{2}\right),
$$

where we used (4.1), and where the bar defines the mean of a random variable. For a homogeneous random field, $\mathscr{C}$ must be a function of $\varrho=\boldsymbol{x}_{1}-\boldsymbol{x}_{2}$. We define $\varrho$ and $\vartheta$ as

$$
\boldsymbol{x}_{1} \cdot \boldsymbol{x}_{2}=R^{2} \cos \vartheta, \quad \varrho=|\varrho|=\left|\boldsymbol{x}_{1}-\boldsymbol{x}_{2}\right|=\sqrt{2} R \sqrt{1-\cos \vartheta} .
$$

It is easy to show that, for a statistically isotropic field on a sphere, i.e. for $\mathscr{C}(\vartheta)$,

$$
\overline{\int_{\ell_{1} m_{1}} \int_{\ell_{2} m_{2}}}=C_{\ell_{1}} \delta_{\ell_{1} \ell_{2}} \delta_{m_{1} m_{2}} \text {. }
$$

for some coefficients $C_{\ell_{1}}$. Indeed, equation (4.6) becomes

$$
\mathscr{C}=\sum_{\ell=0}^{\infty} C_{\ell} \sum_{m=-\ell}^{\ell} Y_{\ell m}\left(\theta_{1}, \phi_{1}\right) Y_{\ell m}\left(\theta_{2}, \phi_{2}\right)=\sum_{\ell=0}^{\infty}(2 \ell+1) C_{\ell} P_{\ell}(\cos \vartheta) \equiv \mathscr{C}(\vartheta),
$$

with $P_{\ell}$ the Legendre polynomial of order $\ell$. The second equality above was achieved using the addition theorem for spherical harmonics. As $\vartheta \rightarrow 0$,

$$
\mathscr{C}(0)=\overline{f^{2}}=\sum_{\ell=0}^{\infty}(2 \ell+1) C_{\ell} .
$$

Identifying $\overline{f^{2}}$ with the spatial average of $f^{2}$ over the sphere, and using Parseval's theorem,

$$
\overline{f^{2}}=\frac{1}{4 \pi} \oiint_{\Omega} f(R, \theta, \phi)^{2} \mathrm{~d} \Omega=\sum_{\ell=0}^{\infty} \sum_{m=-\ell}^{\ell} f_{\ell m}^{2} .
$$

Combining $(4.10)$ and $(4.11)$ :

$$
C_{\ell}=\frac{1}{2 \ell+1} \sum_{m=-\ell}^{\ell} f_{\ell m}^{2}
$$

These are the coefficients of the so-called angular power spectrum of the random field $f$, and they will be used to characterize turbulent statistics extrapolated on spherical surfaces.

Conversely, to construct a statistically homogeneous, isotropic field $f(R, \theta, \phi)$ on a sphere, one must choose its coefficients $f_{i m}$ such that $(4.12)$ is valid for a specified $C_{\ell}$. For example, we propose

$$
f_{\ell m}=\sqrt{(2 \ell+1) C_{\ell}} \frac{\cos \left(2 \pi \omega_{\ell}^{m}\right)}{\sqrt{\sum_{j=-\ell}^{\ell} \cos \left(2 \pi \omega_{\ell}^{j}\right)^{2}}} .
$$

where $\omega_{i}^{j}$ are randomly generated numbers in $[0,1]$. This methodology is used to set up the initial interface perturbation as a $(\theta, \phi)$-isotropic field. 


\subsection{Relation to the energy-spectrum function $E(k)$}

In homogeneous turbulence, the two-point velocity correlation and the velocityspectrum tensor form a Fourier-transform pair:

$$
\Phi_{i j}(\boldsymbol{k})=\frac{1}{(2 \pi)^{3}} \iiint_{-\infty}^{\infty} \mathscr{R}_{i j}(\varrho) \mathrm{e}^{-i \boldsymbol{k} \cdot \varrho} \mathrm{d} \varrho . \quad \mathscr{R}_{i j}(\varrho)=\iiint_{-\infty}^{\infty} \Phi_{i j}(\boldsymbol{k}) \mathrm{e}^{i k \cdot \varrho} \mathrm{d} \boldsymbol{k},
$$

where $\mathscr{R}_{i j}(\varrho) \equiv \overline{u_{i}\left(\boldsymbol{x}_{1}\right) u_{j}\left(\boldsymbol{x}_{2}\right)}$. The energy-spectrum function $E(k)$ is defined by contraction of $\Phi_{i j}$ and integration on a sphere in $\boldsymbol{k}$-space to give

$$
E(k)=\oiint_{\Omega_{k}} \frac{1}{2} \Phi_{i i}(\boldsymbol{k}) \mathrm{d} \Omega_{k}
$$

In isotropic turbulence,

$$
\Phi_{i j}(k)=\frac{E(k)}{4 \pi k^{2}}\left(\delta_{i j}-\frac{k_{i} k_{j}}{k^{2}}\right) .
$$

Since $\Phi_{i i}=E(k) /\left(2 \pi k^{2}\right)$ is a function of the magnitude $k$, its inverse Fourier transform $\mathscr{R}_{i i}$ depends only on the magnitude $\varrho$. For two-point correlations on a sphere, this implies that $\mathscr{R}_{i i}$ only depends on the separation angle $\vartheta$. In the particular case $f=u_{i}$, $\mathscr{R}_{i i}$ corresponds to $\mathscr{C}(\vartheta)$, and from (4.14),

$$
\begin{aligned}
\mathscr{C}(\vartheta) & =\iiint_{-\infty}^{\infty} \frac{E(k)}{2 \pi k^{2}} \mathrm{e}^{i k \cdot \varrho} \mathrm{d} \boldsymbol{k}, \quad \text { with } \boldsymbol{k} \cdot \varrho=k \varrho \cos \theta_{k} \\
& =\int_{0}^{\infty} \frac{E(k)}{2 \pi}\left(\oiint_{\Omega_{k}} \mathrm{e}^{t k \varrho \cos \theta_{k}} \mathrm{~d} \Omega_{k}\right) \mathrm{d} k=2 \int_{0}^{\infty} E(k) \frac{\sin (k \varrho)}{k \varrho} \mathrm{d} k .
\end{aligned}
$$

Since the Legendre polynomials represent an orthogonal basis of functions satisfying

$$
\int_{-1}^{1} P_{\ell}(\xi) P_{m}(\xi) \mathrm{d} \xi=\frac{2}{2 \ell+1} \delta_{\ell m},
$$

then (4.9) implies that

$$
C_{\ell}=\frac{1}{2} \int_{0}^{\pi} \mathscr{C}(\vartheta) P_{\ell}(\cos \vartheta) \sin \vartheta \mathrm{d} \vartheta=\int_{0}^{\infty} E(k) \mathscr{I}_{\ell}(k R) \mathrm{d} k,
$$

where we have used the result of (4.17), and where

$$
\begin{aligned}
\mathscr{I}_{\ell}(k R) & =\int_{0}^{\pi} P_{\ell}(\cos \vartheta) \frac{\sin (\sqrt{2} k R \sqrt{1-\cos \vartheta})}{\sqrt{2} k R \sqrt{1-\cos \vartheta}} \sin \vartheta \mathrm{d} \vartheta \\
& =\int_{-1}^{1} P_{\ell}(x) \frac{\sin (\sqrt{2} k R \sqrt{1-x})}{\sqrt{2} k R \sqrt{1-x}} \mathrm{~d} x \\
& =\sum_{j=0}^{\ell} \frac{4 \beta_{\ell j}}{(2 k R)^{2 j+2}} \int_{0}^{2 k R} y^{2 j} \sin y \mathrm{~d} y \\
& =\sum_{j=0}^{\ell} \frac{2 \beta_{\ell j}}{1+j^{1}} F_{2}\left(j+1 ; \frac{3}{2}, j+2 ;-k^{2} R^{2}\right),
\end{aligned}
$$


where ${ }_{1} F_{2}$ is the generalized hypergeometric function. The third equality was obtained by using the change of variable $y \leftrightarrow \sqrt{2} k R \sqrt{1-x}$ and writing $P_{\ell}$ as the following sum:

$$
P_{\ell}(x)=\sum_{j=0}^{\ell} \beta_{\ell j}\left(\frac{1-x}{2}\right)^{j} \text {, with } \beta_{\ell j}=\left(\begin{array}{l}
\ell \\
j
\end{array}\right)\left(\begin{array}{c}
-\ell-1 \\
j
\end{array}\right) .
$$

Equation (4.19) then becomes

$$
C_{\ell}=\sum_{j=0}^{\ell} \frac{2 \beta_{\ell j}}{j+1} \int_{0}^{\infty} E(k)_{1} F_{2}\left(j+1 ; \frac{3}{2}, j+2 ;-k^{2} R^{2}\right) \mathrm{d} k .
$$

Assume now a power-law energy-spectrum function $E(k)=E_{0}\left(k / k_{0}\right)^{-\alpha}$, with $\alpha>0$ :

$$
\begin{aligned}
& \int_{0}^{\infty} E(k)_{1} F_{2}\left(j+1 ; \frac{3}{2}, j+2 ;-k^{2} R^{2}\right) \mathrm{d} k \\
& =-2^{\alpha} \Gamma(-\alpha) \sin \left(\frac{\pi}{2} \alpha\right)\left(k_{0} R\right)^{\alpha} \frac{E_{0}}{R} \frac{j+1}{2 j+\alpha+1},
\end{aligned}
$$

and (4.22) becomes

$$
C_{\ell}=-2^{\alpha+1} \Gamma(-\alpha) \sin \left(\frac{\pi}{2} \alpha\right)\left(k_{0} R\right)^{\alpha} \frac{E_{0}}{R} \sum_{j=0}^{\ell} \frac{\beta_{\ell j}}{2 j+\alpha+1} .
$$

Using the definition (4.21) of $\beta_{\ell j}$, we finally obtain after some simplifications

$$
C_{\ell}=-2^{\alpha} \frac{\Gamma(-\alpha) \Gamma\left(\frac{1+\alpha}{2}\right)}{\Gamma\left(\frac{1-\alpha}{2}\right)} \sin \left(\frac{\pi}{2} \alpha\right)\left(k_{0} R\right)^{\alpha} \frac{E_{0}}{R} \frac{\Gamma\left(\ell-\frac{\alpha}{2}+\frac{1}{2}\right)}{\Gamma\left(\ell+\frac{\alpha}{2}+\frac{3}{2}\right)} .
$$

In the limit $\ell \gg 1$, we can then show that an energy-spectrum function $E(k) \sim k^{-\alpha}$ leads to an angular power spectrum $C_{\ell} \sim \ell^{-(\alpha+1)}$. More precisely,

$$
C_{\ell}=-2^{\alpha} \frac{\Gamma(-\alpha) \Gamma\left(\frac{1+\alpha}{2}\right)}{\Gamma\left(\frac{1-\alpha}{2}\right)} \sin \left(\frac{\pi}{2} \alpha\right) \frac{1}{k_{0} R} \frac{E_{0}}{R}\left(\frac{\ell}{k_{0} R}\right)^{-(\alpha+1)} .
$$

This relates to a two-dimensional radial power spectrum $E^{\text {Plan }}(\kappa)$, with $\kappa^{2}=k_{x}^{2}+k_{v}^{2}$, that characterizes a statistically isotropic turbulent field on a $(x, y)$-plane, for which an energy-spectrum function $E(k) \sim k^{-\alpha}$ would give $E^{\text {Plan }} \sim \kappa^{-\alpha}$. First observe that the harmonic modes in planar and spherical geometry are best related via the eigenvalue of the two-dimensional part of the Laplacian operator, i.e. $\kappa^{2}$ in planar geometry and $\ell(\ell+1) / R^{2}$ in spherical geometry. The spherical harmonic modes are not simple waves, so one cannot define an appropriate wavelength for them. However, one can use the connection defined by the Laplacian eigenvalues to refer to the 'wavenumber' of the modes: $\kappa^{2}=\ell(\ell+1) / R^{2}$. For $\ell \gg 1$ and at positions on the sphere not too close to the pole, a given spherical harmonic $Y_{\ell}^{m}$ can be approximately represented as a superposition of planar modes whose wavevectors point in different directions but are of magnitude $\kappa \simeq \ell / R$. In this limit, $\ell C_{\ell} \sim \kappa^{-\alpha}$ (see (4.10)), and we naturally recover the scaling of the planar power spectrum. Intuitively, the small-scale turbulent modes do not feel the curvature of the sphere. 


\subsection{Results}

At a given time $t$, consider LES data, $f\left(r_{c}, \theta_{i}, \phi_{j} ; t\right)$, interpolated on the sphere of radius $r=r_{c}(t)$. We first compute the perturbed field $f^{\prime}$ from its spherical surface average, from which the coefficients $f_{\ell m}$ are derived, using (4.5). For example, for $m>0$,

$$
\begin{aligned}
f_{\ell m} & =\frac{1}{4 \pi} \oiint_{\Omega} f^{\prime}\left(r_{c}, \theta, \phi ; t\right) Y_{\ell m}(\theta, \phi) \mathrm{d} \Omega, \quad \text { where } \mathrm{d} \Omega=\sin \theta \mathrm{d} \theta \mathrm{d} \phi \\
& \simeq \frac{\Delta \theta \Delta \phi}{4 \pi} \sum_{i=0}^{N_{\theta}} \sum_{j=0}^{N_{\phi}-1} f^{\prime}\left(r_{c}, \theta_{i}, \phi_{j} ; t\right) \underbrace{N_{\ell m} P_{\ell}^{m}\left(\cos \theta_{i}\right) \cos \left(m \phi_{j}\right)}_{Y_{\ell m}\left(\theta_{i}, \phi_{j}\right) \text { for } m>0} \sin \theta_{i},
\end{aligned}
$$

where $\Delta \theta=\Delta \phi=\Delta / r_{c}$. Rearranging,

$$
f_{\ell m}=\frac{\Delta^{2}}{4 \pi r_{c}^{2}} \sum_{i=0}^{N_{\theta}} N_{\ell m} P_{i}^{n}\left(\cos \theta_{i}\right) \sin \theta_{i} \sum_{j=0}^{N_{\phi}-1} f^{\prime}\left(r_{c}, \theta_{i}, \phi_{j} ; t\right) \cos \left(m \phi_{j}\right) .
$$

Therefore, at a given time $t$ and for a given field $f$, the calculation of each coefficient $f_{\ell m}$, i.e. for a given couple $(\ell, m)$, requires $N_{\theta}\left(r_{c}\right)=\left\lceil\pi r_{c} / \Delta\right\rceil$ evaluations $P_{\ell}^{m}\left(\cos \theta_{i}\right)$, which can be particularly expensive for large $\ell$, even when using a recursion formula. Because there presently exists no truly fast spherical harmonic transforms, we precompute once the $P_{\ell}^{m}$ on a very fine $\theta$-grid. The $P_{\ell}^{m}\left(\cos \theta_{i}\right)$ are then obtained using an interpolation onto the grid of $N_{\theta}\left(r_{c}\right)$ subdivisions at time $t$. The coefficients $C_{\ell}$ of the angular power spectrum are then evaluated following (4.12). For a comparison with the $-5 / 3$ Kolmogorov scaling, we actually plot $\ell C_{\ell}$ as well as the slope $\ell^{-5 / 3}$ (as explained at the end of $\S 43$ ). To be able to compare in the same figure the spectra of a given field at various times, and therefore for different values of $r_{c}$, we plot $\ell C_{\ell}$ as a function of the dimensionless wavenumber $n_{k}=(L / 2 \pi) k$, where we arbitrarily define $k=\ell / r_{c}$ and where $L$ is the size of the computational domain in each Cartesian direction (we recall that the domain is a cube of edge length $L=(\pi / 2) R_{0}$, see $\S 3.1$ of Part 1). The maximum resolved wavenumber $\ell_{\max }$ is such that $\ell_{\max } \sim k_{\max } r_{c}=\pi r_{c} / \Delta=$ $N_{\theta}\left(r_{c}\right)$. In other words, $n_{k_{\max }}=N / 2$, where $N=L / \Delta=512$ would be the number of subdivisions in each Cartesian direction if the grid was refined to the maximum level everywhere.

The resolved angular power spectra of the kinetic energy $u_{i} u_{i}$ and density field $\rho$ are shown in figures 14 and 15 , respectively. For each field, we have chosen three different times of observation: $t=0.5 t_{\text {Resl }}, 2 t_{\text {Res } 1}$ and $3.5 t_{\text {Resl }}$. Consider first figure 14. The initial perturbation signature, characterized by a Gaussian spectrum, is still apparent at early times. The first reshock event has deposited a large amount of energy, which is visible by an increase of $\ell C_{\ell}$ from $t=0.5 t_{\text {Res } 1}$ to $2 t_{\text {Res } 1}$. In the late-time decaying turbulence phase of the flow, a wide inertial subrange develops at the high wavenumbers, with a $-5 / 3$ scaling of the Kolmogorov type, as observed in simulations of planar RM and RT mixing (Hill, Pantano \& Pullin 2006; Chung \& Pullin 2010). A similar scaling was also reached for a spherically imploding mixing layer at higher convergence ratio $R_{0} / r_{c} \approx 8$ and comparable $\ell_{0}$ (Thomas \& Kares 2012). We conclude that, even though the convergence ratio reached here is rather modest $\left(R_{0} / r_{c} \approx 2\right.$ in both the heavy-light and light-heavy configurations; cf. Part 1$)$, the large wavenumbers do not feel the effect of the mixing-layer curvature and that spherical turbulent mixing locally resembles its planar analogue. These conclusions 

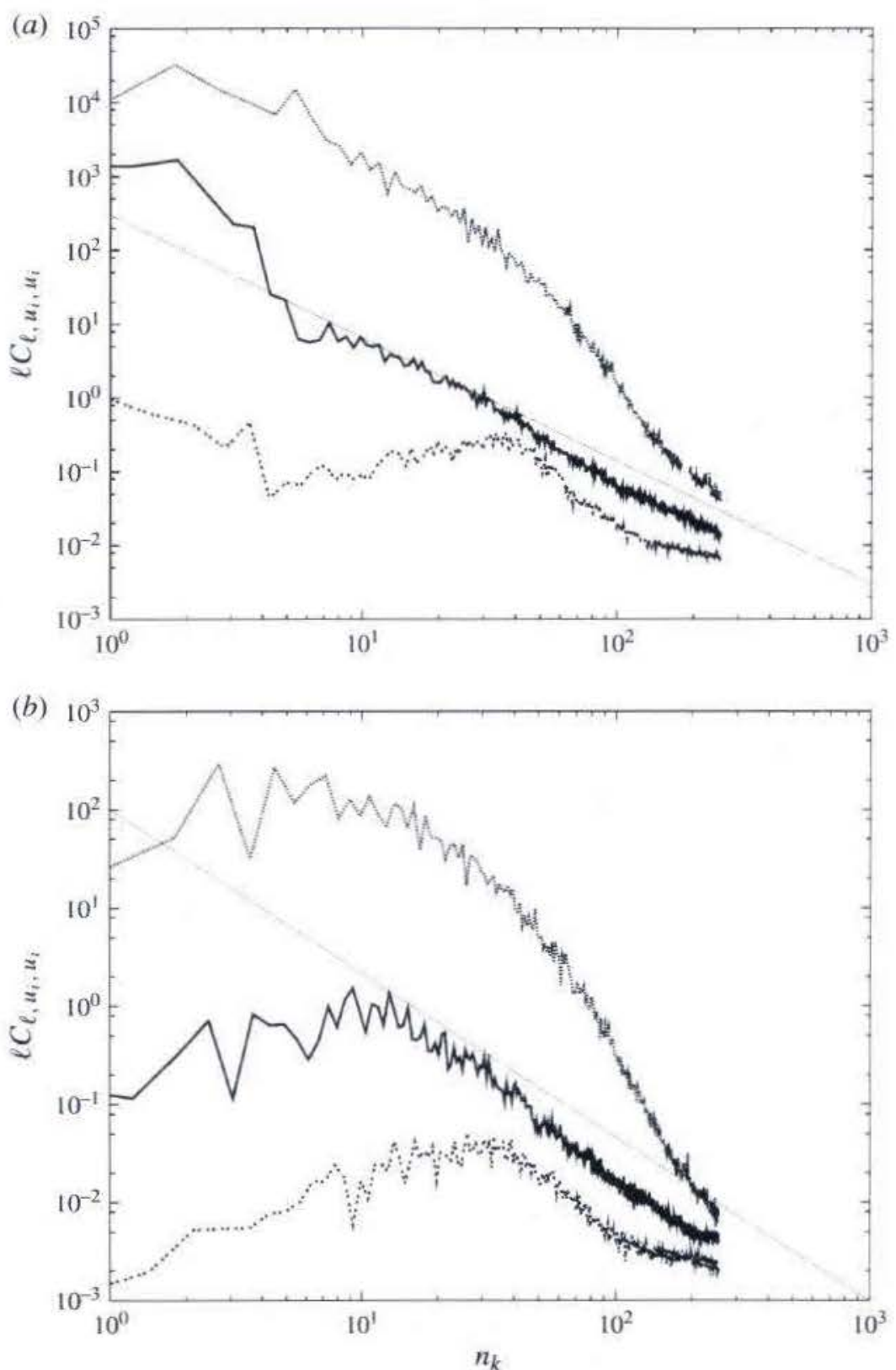

FIGURE 14. Velocity (all components) angular power spectra at three different times, taken at $r=r_{c}(t): t=0.5 t_{\text {Res } 1}$ (dashed line), $2 t_{\text {Res } 1}$ (dotted line), and $3.5 t_{\text {Res } 1}$ (solid line): (a) air $\rightarrow \mathrm{SF}_{6}$ and $(b) \mathrm{SF}_{6} \rightarrow$ air. The $\ell^{-5 / 3}$ power law is represented by a thin straight line. All computed wavenumbers shown and $n_{k_{\max }}=256$.

equally apply to light-heavy and heavy-light spherical mixing. Figure 15 confirms the previous observations but the inertial subrange is contaminated by the presence of aliased modes piling up at high wavenumbers (especially in the heavy-light configuration).

We recall that, for the accurate computation of compressible turbulent mixing, (i) weighted essentially non-oscillatory (WENO) was not used within the mixing region and (ii) no explicit filtering of any kind was performed, as both tend to 

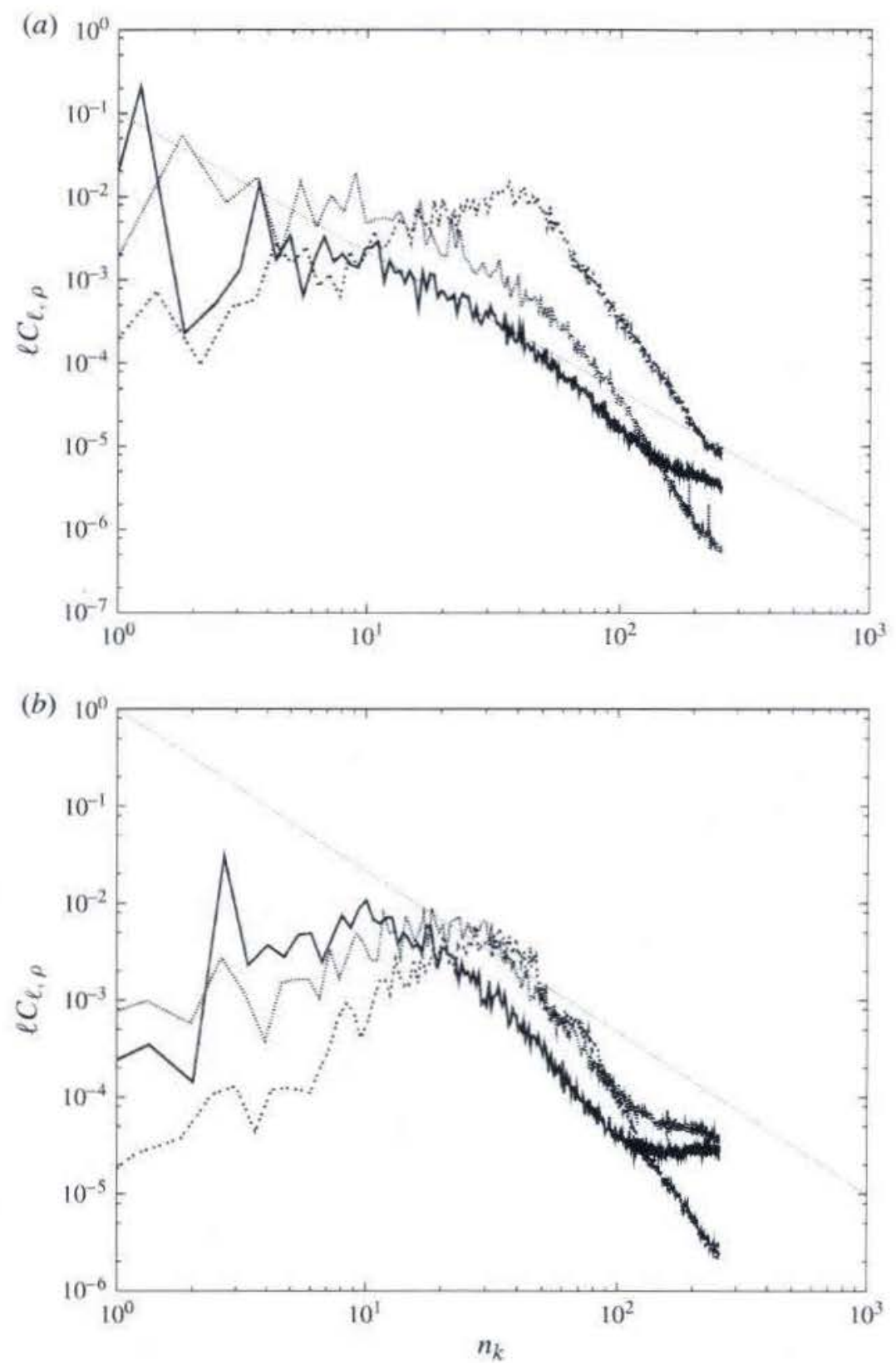

FigURE 15. Density angular power spectra at three different times, taken at $r=r_{c}(t)$ : $t=0.5 t_{\text {Res } 1}$ (dashed line), $2 t_{\text {Res } 1}$ (dotted line) and $3.5 t_{\text {Res } 1}$ (solid line). The $\ell^{-5 / 3}$ power law is represented by a thin straight line. All computed wavenumbers shown and $n_{k_{\max }}=256$ : (a) air $\rightarrow \mathrm{SF}_{6}$ and (b) $\mathrm{SF}_{6} \rightarrow$ air.

artificially remove energy from the highest resolved wavenumbers (Johnsen et al. 2010). The use of skew-symmetric-type discretizations (Honein \& Moin 2004) together with bandwidth-optimized centred stencils, such as the present tuned centre-difference (TCD) scheme (Hill \& Pullin 2004), leads in general to good spectral results with minimal accumulation of energy owing to aliasing errors at the high-wavenumber end of the spectrum. However, in the simulation of turbulent mixing in planar (Hill et al. 2006) and cylindrical geometry (Lombardini, Deiterding \& Pullin 2008), aliasing pile-up also appeared to be larger in scalar-type power spectra 
(e.g. for the density, mass fraction and temperature fields) than in kinetic energy spectra, especially at late times. Kosovic, Pullin \& Samtaney (2002) compared the stretched-vortex SGS model presently employed with a nonlinear SGS model for the simulation of decaying compressible turbulence, both models accounting for important effects of the backscatter of TKE. A low-numerical dissipation centred (compact) differencing scheme was still employed (Lele 1992) and the advective term discretized using a skew-symmetric form without any form of dealiasing. Kosovic et al. (2002) observed similar spectral behaviour at high wavenumbers. Linear Smagorinksy-type SGS models with a positive eddy viscosity might offer better stability results but are absolutely dissipative and do not consider energy backscatter. In the presence of periodic boundaries, dealiasing using the 3/2-rule led to improved spectral results in planar RT-like mixing (Chung \& Pullin 2010). An alternative for the present geometry would consist in dealiasing in the spherical wavenumber space at every time step, but this supposes an entirely new mathematical formulation and numerical implementation.

\section{Summary of results}

While Part 1 of this study focused on the growth of a spherical mixing layer impacted by a concentric implosion, presently we have investigated various turbulence statistics for both light-heavy and heavy-light mixing. Owing to the symmetry of the flow, we have calculated various spherical surface-averaged statistics. In particular, we have developed a spectral analysis for statistically isotropic turbulent fields on spherical surfaces. We recapitulate the following findings.

(i) If molecular mixing is seen as a passive chemical equilibrium between light and heavy fluids, the total molecular mixing product $\mathscr{P}(t)$ and the maximum molecular mixing product $\delta(t)$ indicated effective competition between the mixing activity and the growth of large, entrained eddies within the layer. The ratio $\Theta=\mathscr{P} / \delta$ saturates at a late-time value $\approx 0.8$ in both light-heavy and heavy-light configurations, but the history of $\Theta(t)$ strongly depends on the configuration.

(ii) Because variable-density turbulent mixing arises from the strong coupling between velocity and density fluctuations, we have looked at both the turbulent intensity $\varepsilon_{\rho}=\left\langle\rho^{\prime 2}\right\rangle^{1 / 2} /\langle\rho\rangle$ at the mixing-layer centre, also called effective Atwood ratio $A_{e}(t)$, and also the density self-correlation $b(r, t)$, whose departure from $\varepsilon_{\rho}$ quantifies non-Boussinesq effects. Here $A_{e}(t)$ evolves in the opposite way of $\Theta(t)$. Both light-heavy and heavy-light configurations are characterized by a final value of $A_{e} \approx 0.6 \mathrm{~A}$. Radial profiles of $b$ show a late-time asymmetry of the mixing layer, with a more pronounced peak on the heavy side in the light-heavy mixing. Non-Boussinesq effects appear to be the strongest at the layer edges. In the surface-averaged transport equation for $b$, the production of density fluctuations by specific volume-dilatation correlations is the most dominant term at late times.

(iii) Surface-averaged TKE and turbulent dissipation rate were defined in the context of explicit LES, and helped characterize the late-time decaying turbulence nature of the flow. The present fractions of subgrid TKE and turbulent dissipation relative to their resolved analogues are comparable in trend to those observed in the planar (with reshock) and cylindrical RM flows at comparable incident Mach numbers (Lombardini 2008; Lombardini et al. 2011). At higher Mach numbers, the relative subgrid (resolved) contribution to the TKE (turbulent dissipation) is expected to be even smaller than its resolved (subgrid) counterpart. 
(iv) The turbulent mixing showed stronger anisotropy at large scales than at small scales. The scale-dependence of this anisotropy was assessed by defining directionally dependent Taylor and Kolmogorov turbulent microscales: directional analysis of the Taylor microscale, which is related to the wrinkling of the interfacial isosurface, showed a radial anisotropy of the flow even at late times, which was corroborated by an inspection of the Reynolds stress anisotropy tensor. The radial structure of this tensor across the light-heavy mixing layer differs greatly from the heavy-light one. A small-scale tendency towards isotropy was inferred by inspection of the directional Kolmogorov scales. These features are often observed in planar and cylindrical RM flows (Lombardini 2008; Lombardini et al. 2011), and planar RT mixing as well (Cabot \& Cook 2006).

(v) Turbulent fields were decomposed using the spherical harmonics basis, from which the concept of angular power spectrum was developed in the special case of a statistically isotropic field on a sphere. Kinetic energy and density turbulent spectra obtained at the mixing-layer centre displayed the formation of an inertial subrange approaching the Kolmogorov-like $-5 / 3$ power law at late times, similarly to planar and cylindrical shock-driven mixing at comparable initial perturbation dominant wavelengths and incident Mach numbers. The spectra were obtained from a narrow Gaussian-type perturbation spectrum with a dominant wavelength small compared with the initial radial position of the interface. The corresponding dominant wavenumber $\ell_{0}$ is comparable to the $\ell=30$ mode asymmetry employed in the ICF implosion simulations of Thomas \& Kares (2012) who obtained a similar $-5 / 3$ power law around the third reshock time. A convergence ratio of 8 for the pusher/gas interface was achieved in their simulations. Like Thomas \& Kares (2012), the evidence of fully developed turbulence at rather low convergence ratios (compared with the high convergence ratio of 30 for the National Ignition Facility ignition target) could be significant for ICF applications as it demonstrates how 'small-to-moderate' $\ell$ mode asymmetry can yield ignition failure as an additional scenario to the usual assumption that degradation is dominated by RT interfacial instabilities of 'large' $\ell$ (i.e. typically $>100)$ modes.

To summarize, heavy-light and light-heavy mixing differ from each other in the following measures: evolutions of $\mathscr{P}(t), \delta(t), A_{e}(t)$ and $\Theta(t)$; and late-time radial profiles of $b(r, t)$ and $B_{r r}(r, t)$. Although heavy-light and light-heavy layers achieve similar convergence ratios and $-5 / 3$ power law for the turbulent spectra, the mixing evolution for each configuration is strongly influenced by their particular RT-unstable history.

\section{Acknowledgement}

This work has been supported in part by the Department of Energy under subcontract no. DE-AC52-06NA25396.

\section{Appendix A. Spherical surface-averaged turbulent budgets}

RANS modelling of turbulent mixing in variable-density flows is based on the turbulent transport equations of $b, a_{i}$ and $\mathscr{K}$. Besnard et al. (1992) derived these equations in planar geometry and we extend them to flows with a spherical symmetry. 


\section{A.1. Density self-correlation equation}

Starting from the continuity equation, and observing that $b=-\left\langle\rho^{\prime} v^{\prime}\right\rangle=\langle\rho\rangle\langle v\rangle-1$, with $v=1 / \rho$, we obtain

$$
\begin{aligned}
& \underbrace{\frac{\partial b}{\partial t}}_{(b \mathscr{U})}+\underbrace{\tilde{u}_{r} \frac{\partial b}{\partial r}}_{(b \mathrm{O})}=\underbrace{2 a_{r} \frac{\partial b}{\partial r}}_{(b \mathrm{I})} \underbrace{-2 \frac{(1+b)}{\langle\rho\rangle} a_{r} \frac{\partial\langle\rho\rangle}{\partial r}}_{(b \mathrm{II})}+\underbrace{\frac{\langle\rho\rangle}{r^{2}} \frac{\partial}{\partial r}\left(r^{2} \frac{\left\langle\rho^{\prime} v^{\prime} u_{r}^{\prime}\right\rangle}{\langle\rho\rangle}\right)}_{(b \mathrm{mI})} \\
& +\underbrace{2\langle\rho\rangle\left(\left\langle v^{\prime} \nabla \cdot \boldsymbol{u}^{\prime}\right\rangle+\frac{1}{r}\left\langle v^{\prime} \cot \theta\right\rangle\right)}_{(b \mathrm{IV})},
\end{aligned}
$$

where

$$
\left\langle v^{\prime} \nabla \cdot \boldsymbol{u}^{\prime}\right\rangle+\frac{\left\langle v^{\prime} \cot \theta\right\rangle}{r}=\langle v \nabla \cdot \boldsymbol{u}\rangle-\frac{\langle v\rangle}{r^{2}} \frac{\partial}{\partial r}\left(r^{2}\left\langle u_{r}\right\rangle\right) .
$$

The convection terms $(b \mathrm{O})$ and $(b \mathrm{I})$ redistribute $b$ across the mixing layer, from the heavy side to the light side and vice versa. Term ( $b$ II) represents the production of $b$ by the mean density gradient, term ( $b$ III) the turbulent transport towards the edges of the mixing layer responsible for the spreading of the $b$-profile, and term $(b$ IV) the production of $b$ by specific volume-dilatation correlations. Terms $(b \mathrm{I})$ and $(b \mathrm{II})$ represents a feedback from the $a_{r}$-equation presented below.

\section{A.2. Turbulent mass flux equation}

Starting from the Favre-filtered momentum equation governing the conservation of resolved-scale momentum $\rho u$, we derive a surface-averaged transport equation for the turbulent mass flux $a_{r}=\left\langle\rho^{\prime} u_{r}^{\prime}\right\rangle /\langle\rho\rangle$ in the radial direction:

$$
\begin{aligned}
& \underbrace{\frac{\partial\left(\langle\rho\rangle a_{r}\right)}{\partial t}}_{(a, \mathscr{U})}+\underbrace{\left\{[\boldsymbol{\nabla} \cdot(\langle\rho\rangle \tilde{\boldsymbol{u}} \otimes \boldsymbol{a})] \cdot \boldsymbol{e}_{r}-\frac{1}{r}\langle\rho\rangle \tilde{u}_{\theta} a_{r} \cot \theta\right\}}_{\left(a_{r} \mathrm{O}\right)} \\
& =\underbrace{b \frac{\partial\langle p\rangle}{\partial r}}_{(a, \mathrm{I})} \underbrace{-b\left[(\nabla \cdot\langle\boldsymbol{\sigma}\rangle) \cdot \boldsymbol{e}_{r}-\frac{1}{r}\left\langle\sigma_{\theta r}\right\rangle \cot \theta\right]}_{\left(a_{r} \text { III }\right)}+\underbrace{\langle\rho\rangle\left\langle v^{\prime} \frac{\partial p^{\prime}}{\partial r}\right\rangle}_{\left(a_{r} \text { III }\right)} \\
& \underbrace{-\langle\rho\rangle\left[\left\langle v^{\prime}\left(\boldsymbol{\nabla} \cdot \boldsymbol{\sigma}^{\prime}\right) \cdot \boldsymbol{e}_{r}\right\rangle+\frac{1}{r}\left\langle\sigma_{\theta r}\right\rangle\left\langle v^{\prime} \cot \theta\right\rangle\right]}_{(a, \mathrm{IV})} \\
& \underbrace{-\langle\rho\rangle[\boldsymbol{a} \cdot \boldsymbol{\nabla}(\widetilde{\boldsymbol{u}}-\boldsymbol{a})] \cdot \boldsymbol{e}_{r}}_{\left(a_{r} \mathrm{~V}\right)}+\underbrace{\left(\frac{\left\langle\rho^{\prime} u_{r}^{\prime} u_{r}^{\prime}\right\rangle-R_{r r}}{\langle\rho\rangle}\right) \frac{\partial\langle\rho\rangle}{\partial r}}_{\left(a_{r} \mathrm{VD}\right)} \\
& +\underbrace{\langle\rho\rangle\left\{[\nabla \cdot(\boldsymbol{a} \otimes \boldsymbol{a})] \cdot \boldsymbol{e}_{r}-\frac{1}{r} a_{\theta} a_{r} \cot \theta\right\}}_{\left(a_{r} \mathrm{VII}\right)}
\end{aligned}
$$




$$
\begin{aligned}
& \underbrace{-\left\{\left[\boldsymbol{\nabla} \cdot\left\langle\rho^{\prime} \boldsymbol{u}^{\prime} \otimes \boldsymbol{u}^{\prime}\right\rangle\right] \cdot \boldsymbol{e}_{r}-\frac{1}{r}\left\langle\rho^{\prime} u_{\theta}^{\prime} u_{r}^{\prime}\right\rangle \cot \theta\right\}}_{\left(a_{r} \mathrm{VIII}\right)}, \\
& \underbrace{-\langle\rho\rangle\left(\left\langle u_{r}^{\prime} \nabla \cdot \boldsymbol{u}^{\prime}\right\rangle+\frac{1}{r}\left\langle u_{r}^{\prime} \cot \theta\right\rangle\left\langle u_{\theta}\right\rangle\right)}_{\left(a_{r} \mathrm{IX}\right)},
\end{aligned}
$$

where $\sigma_{i j}$ are the (resolved + subgrid) stress tensor components, and, for any vectors $c$ and $d$,

$$
[\nabla \cdot(c \otimes d)] \cdot e_{r}-\frac{1}{r} c_{\theta} d_{r} \cot \theta=\frac{1}{r^{2}} \frac{\partial}{\partial r}\left(r^{2} c_{r} d_{r}\right)-\frac{c_{\theta} d_{\theta}+c_{\phi} d_{\phi}}{r} .
$$

Terms $\left(a_{r} \mathrm{O}\right)$ and $\left(a_{r} \mathrm{VII}\right)$, of opposite sign and close magnitude, transport the turbulent mass flux from the heavy to light fluid and vice versa. The transport part of $\left(a_{r}\right.$ VIII) spreads the turbulent mass flux by moving it from the interior towards the edges of the mixing layer. Term $\left(a_{r}\right.$ III $)+\left(a_{r}\right.$ IV $)$ and $\left(a_{r}\right.$ VI $)$ are production terms. Term $\left(a_{r} \mathrm{I}\right)+\left(a_{r} \mathrm{II}\right)$ is also a production term, which depends on the mix state through $b$. Term $\left(a_{r} \mathrm{IX}\right)$, which involves the radial velocity-dilatation correlation, is expected to be negligible in weakly compressible turbulence.

\section{A.3. TKE equation}

From the momentum equation, we derive the surface-averaged transport equation for the TKE $\mathscr{K} \equiv R_{k k} /(2\langle\rho\rangle)$ :

$$
\begin{aligned}
& \underbrace{\frac{\partial(\langle\rho\rangle \mathscr{K})}{\partial t}}_{(\mathscr{K} \mathscr{U})}+\underbrace{\frac{\partial\left(\langle\rho\rangle \mathscr{K} \tilde{u}_{r}\right)}{\partial r}}_{(\mathscr{K} \mathrm{O})}=\underbrace{-\left[\boldsymbol{R}: \nabla \tilde{\boldsymbol{u}}+\frac{1}{r}\left(R_{\theta \phi} \tilde{u}_{\phi}-R_{\phi \phi} \tilde{u}_{\theta}\right) \cot \theta\right]}_{(\mathscr{K} \mathrm{D})} \\
& \underbrace{-\frac{1}{r^{2}} \frac{\partial}{\partial r}\left(r^{2}\left\langle p^{\prime} u_{r}^{\prime}\right\rangle\right)}_{(\mathscr{H} \mathrm{II})}+\underbrace{\left(a_{r} \frac{\partial\langle p\rangle}{\partial r}-\frac{1}{r}\langle p \cot \theta\rangle a_{\theta}\right)}_{(\mathscr{K} \mathrm{II})} \\
& +\underbrace{\left\langle p^{\prime} \boldsymbol{\nabla} \cdot \boldsymbol{u}^{\prime}\right\rangle}_{(\mathscr{X} \mathrm{VV})} \underbrace{-\frac{1}{2 r^{2}} \frac{\partial}{\partial r}\left(r^{2}\left\langle\rho u_{i}^{\prime \prime} u_{i}^{\prime \prime} u_{r}^{\prime \prime}\right\rangle\right)}_{(\mathscr{K} \mathrm{V})} \underbrace{-\left\langle\boldsymbol{\sigma}: \nabla \boldsymbol{u}^{\prime}\right\rangle}_{(\mathscr{K} \mathrm{VI})} \\
& +\underbrace{\frac{1}{r^{2}} \frac{\partial}{\partial r}\left(r^{2}\left\langle\sigma_{i r} u_{i}^{\prime}\right\rangle\right)}_{(\mathscr{K} \text { VII })} \underbrace{-\langle a \cdot(\nabla \cdot \sigma)\rangle}_{(\mathscr{X} \text { VIII })} \text {, }
\end{aligned}
$$

where

$$
\begin{aligned}
\boldsymbol{R}: \nabla \widetilde{\boldsymbol{u}}+\frac{1}{r}\left(R_{\theta \phi} \widetilde{u}_{\phi}-R_{\phi \phi} \widetilde{u}_{\theta}\right) \cot \theta= & R_{r r} \frac{\partial \widetilde{u}_{r}}{\partial r}+R_{r \theta}\left(\frac{\partial \widetilde{u}_{\theta}}{\partial r}-\frac{\widetilde{u}_{\theta}}{r}\right) \\
& +R_{\theta \theta} \frac{\widetilde{u}_{r}}{r}+R_{r \phi}\left(\frac{\partial \widetilde{u}_{\phi}}{\partial r}-\frac{\widetilde{u}_{\phi}}{r}\right)+R_{\phi \phi} \frac{\widetilde{u}_{r}}{r} .
\end{aligned}
$$

The transport terms $(\mathscr{K} \mathrm{O}),(\mathscr{K}$ II $),(\mathscr{K} \mathrm{V})$ and $(\mathscr{K}$ VII $)$ redistribute the TKE inside the mixing layer. In particular, term $(\mathscr{K} \mathrm{O})$ is responsible for the asymmetry of the 
TKE profile by transporting TKE from heavy to light sides; term $(\mathscr{K} \mathrm{V})$ spreads the TKE profile by transporting TKE from the interior to the edges of the mixing layer, while $(\mathscr{K}$ II) has the opposite effect. Term $(\mathscr{K}$ I) represents the TKE exchange between the mean flow and the turbulent fluctuations. The viscous dissipation term ( $\mathscr{K} \mathrm{VI}$ ) is expected to remain symmetrical, as non-Boussinesq effects primarily affect the large-scale mean pressure gradient and velocity. Term ( $\mathscr{K}$ IV) is expected to vanish in weakly compressible turbulence. The TKE equation is coupled to the $a_{r}$-equation through the terms $(\mathscr{K}$ III) and ( $\mathscr{K}$ VIII).

Equation (A.5) is a transport equation for the surface-averaged resolved TKE, not the surface-averaged resolved + subgrid TKE. Therefore, the dissipation rate ( $\mathscr{K}$ VI), which led to the definitions $(3.1 c, d)$, represents the dissipation rate of resolved TKE. This is acceptable because the TKE is given for the most part by its resolved fraction (figure 6). To be rigorous, one could actually derive a surface-averaged equation for the total TKE as follows. First, derive the Favre-filtered (LES) equation for the subgrid TKE $\tau_{i i} / 2 \rho$, e.g. equation (10) of Pomraning \& Rutland (2002); note how this equation naturally introduces extra subgrid correlations to model, in particular the dissipation term (12) in that paper. Second, derive the surface-averaged equation for $\left\langle\tau_{i i}\right\rangle / 2\langle\rho\rangle$. Third, add that last equation to (A 5) to obtain the surface-averaged equation for the total TKE.

\section{REFERENCES}

BALAKRISHNAN, K. \& MENON, S. 2011 Characterization of the mixing layer resulting from the detonation of heterogeneous explosive charges. Flow Turbul. Combust. 87, 639-671.

Besnard, D., Harlow, F. H., Rauenzahn, R. M. \& Zemach, C. 1992 Turbulent transport equations for variable-density turbulence and their relationship to two-fluid models. LANL Tech. Rep. LA-12303-MS.

САВот, W. H. \& СОоK, A. W. 2006 Reynolds number effects on Rayleigh-Taylor instability with possible implications for type Ia supernovae. Nat. Phys. 2 (8), 562-568.

Chung, D. \& Pullin, D. I. 2010 Direct numerical simulation and large-eddy simulation of stationary buoyancy-driven turbulence. J. Fluid Mech. 643, 279-308.

COOK, A. W., САВOT, W. \& MILLER, P. L. 2004 The mixing transition in Rayleigh-Taylor instability. J. Fluid Mech. 511, 333-362.

Glimm, J., Grove, J. W., Zhang, Y. \& Dutta, S. 2002 Numerical study of axisymmetric Richtmyer-Meshkov instability and azimuthal effect on spherical mixing. J. Stat. Phys. 107 (1/2), 241-260.

Gotoh, T., Watanabe, T. \& SUZUKi, Y. 2011 Universality and anisotropy in passive scalar fluctuations in turbulence with uniform mean gradient. J. Turbul. 12, N48.

Hill, D. J., Pantano, C. \& Pullin, D. I. 2006 Large-eddy simulation and multi-scale modeling of Richtmyer-Meshkov instability with reshock. J. Fluid Mech. 557, 29-61.

HiLl, D. J. \& PUllin, D. I. 2004 Hybrid tuned center-difference-WENO method for large eddy simulations in the presence of strong shocks. J. Comput. Phys. 194 (2), 435-450.

HoneIN, A. E. \& MoIN, P. 2004 Higher entropy conservation and numerical stability of compressible turbulence simulations. J. Comput. Phys. 201 (2), 531-545.

Joggerst, C. C., Almgren, A. \& Woostey, S. E. 2010 Three-dimensional simulations of RayleighTaylor mixing in core-collapse supernovae. Astrophys. J. 723, 353-363.

Johnsen, E., Larsson, J., Bhagatwala, A. V., Cabot, W. H., Moin, P., Ol.son, B. J., Rawat, P. S., Shankar, S, K., Siögreen, B., Yee, H. C., Zhong, X. \& Lele, S. K. 2010 Assessment of high-resolution methods for numerical simulations of compressible turbulence with shock waves. J. Comput. Phys. 229, 1213-1237.

Kosovic, B., Pullin, D. I. \& Samtaney, R. 2002 Subgrid-scale modeling for large-eddy simulations of compressible turbulence. Phys. Fluids 14 (4), 1511-1522. 
Lele, S. K. 1992 Compact finite difference schemes with spectral-like resolution. J. Comput. Phys. $103,16-42$.

Livescu, D. \& Ristorcelli, J. R, 2007 Buoyancy-driven variable-density turbulence. J. Fluid Mech. 591, 43-71.

Livescu, D. \& Ristorcell., J. R. 2008 Variable-density mixing in buoyancy-driven turbulence. J. Fluid Mech. 605, 145-180.

LivesCU, D. \& Ristorcell., J. R. 2009 Mixing asymmetry in variable density turbulence. In Advances in Turbulence XII (ed. B. Eckhardt), Springer Proceedings in Physics, vol. 132, pp. 545-548. Springer.

Livescu, D., Ristorcelli, J. R., Gore, R. A., Dean, S. H., Cabot, H. W. \& Cook, A. W. 2009 High Reynolds number Rayleigh-Taylor turbulence. J. Turbul. 10 (13), 1-32.

LOMBARDINI, M. 2008 Richtmyer-Meshkov instability in converging geometries. PhD thesis, California Institute of Technology. http://thesis.library caltech edu/2319/.

Lombardini, M., Deiterding, R. \& Pullin, D. I. 2008 Large-eddy simulation of the Richtmyer-Meshkov instability in a converging geometry. In Quality and Reliability of Large-Eddy Simulations, Proc. of QLES 2007 Intl Symposium (ed. J. Meyers, B. J. Geurts \& P. Sagaut), ERCOFTAC Series, vol. 12, pp. 23-51. Springer.

Lombardini, M., Hill, D. J., Pullin, D. I. \& Meiron, D. I. 2011 Atwood ratio dependence of Richtmyer-Meshkov flows under reshock conditions using large-eddy simulations. J. Fluid Mech. 670, 439-480.

Lombardini, M., Pullin, D. I. \& Meiron, D. I. 2014 Turbulent mixing driven by spherical implosions. Part 1. Flow description and mixing-layer growth. J. Fluid Mech. 748, 85-112.

Meshrov, E. E. 1969 Instability of the interface of two gases accelerated by a shock wave. Sov. Fluid Dyn. 4 (5), 101-108.

OVERHOlt, M. R. \& POPE, S. B. 1996 Direct numerical simulation of a passive scalar with imposed mean gradient in isotropic turbulence. Phys. Fluids 8, 3128-3148.

POMRANING, E. \& RUTLAND, C. J. 2002 Dynamic one-equation nonviscosity large-eddy simulation model. AIAA J. 40 (4), 689-701.

PUlLin, D. I. 2000 A vortex-based model for the subgrid flux of a passive scalar. Phys. Fluids 12 (9), 2311-2319.

RICHTMYeR, R. D. 1960 Taylor instability in shock acceleration of compressible fluids. Commun. Pure Appl. Maths 13, 297-319.

RISTORCELLI, J. R. 2006 Passive scalar mixing: analytic study of time scale ratio, variance, and mix rate. Phys. Fluids 18, 124103.

RistorCElLI, J. R. \& CLARK, T. T. 2004 Rayleigh-Taylor turbulence: self-similar analysis and direct numerical simulations. J. Fluid Mech. 507, 213-253.

TAYLOR, G. I. 1950 The instability of liquid surfaces when accelerated in a direction perpendicular to their planes. Proc. R. Soc. Lond. A 201, 192-196.

THOMAS, V. A. \& KARES, R. J. 2012 Drive asymmetry and the origin of turbulence in an ICF implosion. Phys. Rev. Lett. 109 (7), 075004.

WAChtor, A. J., Grinstein, F. F., DeVore, C. R., Ristorcelli, J. R. \& Margolin, J. G. 2013 Implicit large-eddy simulation of passive scalar mixing in statistically stationary isotropic turbulence. Phys. Fluids 25 (2), 025101.

WARHAFT, Z. \& LUMLEY, J. L. 1978 An experimental study of the decay of temperature fluctuations in grid-generated turbulence. J. Fluid Mech. 88, 659-684.

Welser-Sherrill, L., Haynes, D. A., Mancini, R. C., Cooley, J. H., Tommasini, R., Golovkin, I. E., SHERrill., M. E. \& HAAN, S. W. 2008 Inference of ICF implosion core mix using experimental data and theoretical mix modeling. High Energy. Dens. Phys. 5 (4), 249-257.

YouNGS, D. L. 1994 Numerical simulations of mixing by Rayleigh-Taylor and Richtmyer-Meshkov instabilities. Laser Part. Beams 12, 725-750.

Youngs, D. L. \& Williams, R. J. R. 2008 Turbulent mixing in spherical implosions. Intl J. Numer. Meth. Fluids 56 (8), 1597-1603. 